\title{
On the Semantics of Place/Transition Petri Nets
}

\author{
JOSÉ MESEGUER, ${ }^{1}$ UGO MONTANARI, ${ }^{2}$ and VLADIMIRO SASSONE ${ }^{2}$ \\ ${ }^{1}$ SRI International, Menlo Park, California 94025 \\ ${ }^{2}$ Dipartimento di Informatica, Università di Pisa, Italy
}

Received 1 April 1996

Place/Transition (PT) Petri nets are one of the most widely used models of concurrency. However, they still lack, in our view, a satisfactory semantics: on the one hand the 'token game' is too intensional, even in its more abstract interpretations in terms of nonsequential processes and monoidal categories; on the other hand, Winskel's basic unfolding construction, which provides a coreflection between nets and finitary prime algebraic domains, works only for safe nets. In this paper we extend Winskel's result to PT nets. We start with a rather general category PTNets of PT nets, we introduce a category DecOcc of decorated (nondeterministic) occurrence nets and we define adjunctions between PTNets and DecOcc and between DecOcc and Occ, the category of occurrence nets. The role of DecOcc is to provide natural unfoldings for PT nets, i.e., acyclic safe nets where a notion of family is used for relating multiple instances of the same place.

The unfolding functor from PTNets to Occ reduces to Winskel's when restricted to safe nets; moreover, the standard coreflection between Occ and Dom, the category of finitary prime algebraic domains, when composed with the unfolding functor above, determines a chain of adjunctions between PTNets and Dom.

\section{Introduction}

Petri nets, introduced by C.A. Petri in (Petri 1962) (see also Petri 1973; Reisig 1985), are a widely used model of concurrency. This model is attractive from a theoretical point of view because of its simplicity and because of its intrinsically concurrent nature, and has often been used as a semantic basis on which to interpret concurrent languages (see for example Winskel 1982; Olderog 1987; van Glabbeek and Vaandrager 1987; Degano et al. 1988).

For Place/Transition (PT) nets, having a satisfactory semantics-one that does justice to their truly concurrent nature, yet is abstract enough-remains in our view an unresolved problem. Certainly, many different semantics have been proposed in the literature; we briefly discuss some of them below.

At the most basic operational level we have of course the 'token game'. To account for computations involving many different transitions and for the causal connections between transition events, various notions of process have been proposed (Petri 1977; Goltz and Reisig 1983; Best and Devillers 1987), but process models do not provide a satisfactory semantic denotation for

Research partially supported by ESPRIT Basic Research Action CEDISYS. The first and the third authors have been supported by the US Office of Naval Research Contracts N00014-88-C-0618 and N00014-92-C-0158. The third author also acknowledges funding from EU Human Capital and Mobility grant ERBCHBGCT 920005 and from BRICS, Basic Research in Computer Science, Centre of the Danish National Research Foundation. 
a net as a whole. In fact, they specify only the meaning of single, deterministic computations, while the accurate description of the fine interplay between concurrency and nondeterminism is one of the most valuable features of nets.

Other semantic investigations have capitalized on the algebraic structure of PT nets, first noticed by Reisig (Reisig 1985) and later exploited by Winskel to identify a sensible notion of morphism between nets (Winskel 1984; Winskel 1987). More recently, a different interpretation of the algebraic structure of PT nets in terms of monoidal categories has been proposed in (Meseguer and Montanari 1990). Alternative interesting algebraic/categorical approaches are (Brown and Gurr 1990; Brown et al. 1991; Martí-Oliet and Meseguer 1991; Engberg and Winskel 1993), based on ideas from linear logic, and (Mukund 1992), which provides an account of net behaviours in terms of (step) transition systems.

One particular advantage of the algebraic approaches based on category theory is that they provide useful net combinators, associated to standard categorical constructions such as product and coproduct, which can be used to give a simple account of corresponding compositional operations at the level of a concurrent programming language, such as various forms of parallel and non-deterministic composition (Winskel 1987; Meseguer and Montanari 1990; Brown et al. 1991; Mukund 1992).

A unification of the process-oriented and algebraic viewpoints has recently been proposed in (Degano et al. 1989; see also the related Sassone 1996; Sassone 1995) by showing that the commutative processes (Best and Devillers 1987) of a net $N$ are isomorphic to the arrows of a symmetric monoidal category $\mathcal{T}[N]$. Moreover, they introduced the concatenable processes of $N$-a slight variation of Goltz-Reisig processes (Goltz and Reisig 1983) on which sequential composition is defined - and structured them as the arrows of the symmetric monoidal category $\mathcal{P}[N]$. That would individuate in the category of the symmetric monoidal categories a semantic domain for PT nets. However, in spite of accounting for algebraic and process aspects in a simple unified way, this semantics is still too concrete, and a more abstract semantics—one allowing greater semantic identifications between nets-would be clearly preferable in many applications.

A very attractive formulation for the semantics that we seek would be an adjoint functor assigning an abstract denotation to each PT net and preserving certain compositional properties in the assignment. This is exactly what Winskel has done for the subcategory of safe nets (Winskel 1986). In that work - which builds on the previous important work (Nielsen et al. 1981) - the denotation of a safe net is a Scott domain (Scott 1970), and Winskel shows that there exists a coreflection - a particularly nice form of adjunction—between the category Dom of (coherent) finitary prime algebraic domains and the category Safe of safe Petri nets. Winskel's coreflection factorizes through the chain of coreflections

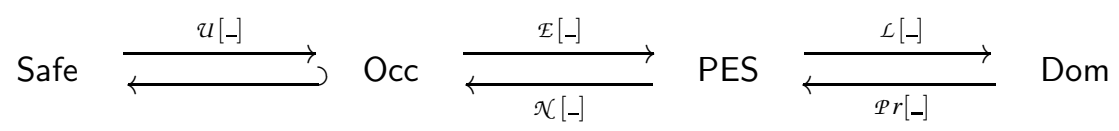

where PES is the category of prime event structures (with binary conflict), which is equivalent to Dom, Occ is the category of occurrence nets (Winskel 1986), $\hookleftarrow$ is the inclusion functor, and the lower arrows are left adjoints.

This construction is completely satisfactory: from the intuitive point of view it gives the 'truly concurrent' semantics of safe nets in the most universally accepted type of model, while from 
the formal point of view the existence of an adjunction guarantees its 'naturality'. In fact, it follows directly from the choice of the denotational models that this semantics is suited to a precise description of causality: every causal interaction among transitions, in principle a historydependent concept, must be resolved to the global, static relations of causality and conflict of event structures. In other words, the unfolding amounts to translating the 'dynamic' structure of nets to the 'static' structure of occurrence nets and event structures, i.e., to 'compiling' transitions to events. Under this translation events are to be thought of as unique occurrences of transitions which bear unique, static causal links to each other. It is worth observing that such a causal semantics is incompatible with the idea of indistinguishability of tokens: different tokens in the same place can carry different causal histories and, therefore, give rise to different events, even when consumed by the same transition. In other words, it could be said that the very purpose of a causal semantics for nets is to distinguish tokens according to their causal history.

The existence of adjoints to the unfolding functors guarantees the adequacy of the semantics. In fact, starting from the natural assumption that a possible denotation of $N$ in PES is an event structure $E S$ together with map $\mathcal{N}[E S] \rightarrow N$ which 'labels' the events with the transitions they correspond to, by the universal property of adjunctions, each possible explanation of $N$ in PES factors uniquely through the one assigned by the unfolding, viz., $\mathcal{N} \mathcal{E} \mathcal{U}[N] \rightarrow N$. Intuitively, this can be interpreted as saying that $\mathcal{E} \mathfrak{U}[N]$ is the 'best possible', the 'most complete' explanation of $N$ in PES, the one which unambiguously subsumes all the (partial) others and, moreover, it is (up to isomorphisms) the unique event structure which enjoys such a property.

Recently, various attempts have been made to extend this chain or, more generally, to identify a suitable semantic domain for PT nets. Among them, we recall (Pratt 1991), where, in order to obtain a model 'mathematically more attractive than Petri nets', a geometric model of concurrency based on $n$-categories as models of higher dimensional automata is introduced, but the modelling power obtained does not seem to be greater than that of ordinary PT nets, though the framework is highly elegantly linked to algebraic topology (Goubault and Jensen 1992; Goubault 1993; see also Cattani and Sassone 1996), (Hoogers et al. 1992), in which the authors give semantics to PT nets in terms of generalized trace languages and discuss how using their work it could perhaps be possible to obtain a concept of unfolding for PT nets; and (Engelfriet 1991), where the unfolding of Petri nets is given in term of a branching process. However, the nets considered in loc. cit. are not general PT nets because their transitions are restricted to have pre and post-sets where all places have no multiplicities. A yet more recent approach is (Hoogers et al. 1993), where the unfolding is explained in terms of a new notion of local event structure. Finally, we would like to cite in this context (Gunawardena 1993).

The present work extends Winskel's approach from safe nets to the category of PT nets. We define the unfoldings of PT nets and relate them by an adjunction to occurrence nets and thereforeexploiting the already existing adjunctions - to prime event structures and finitary prime algebraic domains. The adjunctions so obtained are extensions of the corresponding Winskel's coreflections. We follow strictly the intuitions of (Winskel 1986) discussed above. Of course, here we need to take care of the multiplicities in pre- and post-sets, which, respectively, consume and produce multiple concurrent tokens in the same place. Although such tokens have the same history, they must be distinguished, since, potentially, they give rise to different events, e.g., multiple concurrent activations of the same transition. This is called individual token interpretation in (van Glabbeek and Plotkin 1995). 
The category PTNets that we consider is quite general. Objects are PT nets in which markings may be infinite and transitions are allowed to have infinite pre- and post-sets, but, as usual, with finite multiplicities. The only technical restriction we impose, with respect to the natural extension to nets with infinite markings of the general formulation in (Meseguer and Montanari 1990), is the usual condition that transitions must have non-empty pre-sets. Actually, the objects of PTNets strictly include those of the categories considered in (Winskel 1986; Winskel 1987). Although a technical restriction applies to the morphisms - they are required to map places belonging to the initial marking or to the post-set of the same transition to disjoint multisets- they are still quite general. The category PTNets has initial and terminal objects, and has products and coproducts which model, respectively, the operations of parallel and non-deterministic composition of nets. It is worth remarking that, while coproducts do not exist in the categories of generally marked, non-safe PT nets considered in (Winskel 1987; Meseguer and Montanari 1990), they do in PTNets. In addition, in (Brown et al. 1991) and in (Mukund 1992), where coproducts exist, they seem not to have a neat computational intepretation.

Concerning the organization of the paper, in Section 1 we define the category PTNets and in Section 2 we show that it has products and coproducts. In Section 3 we introduce a new kind of nets, the decorated occurrence nets, which naturally represent the unfoldings of PT nets and can account for the multiplicities of places in transitions. They are occurrence nets in which places belonging to the post-set of the same transition are partitioned into families. Families are used to relate places corresponding in the unfolding to multiple instances of the same place in the original net. When all the families of a decorated occurrence net have cardinality one, we have (a net isomorphic to) an ordinary occurrence net. Therefore, Occ is (isomorphic to) a full subcategory of DecOcc, the category of decorated occurrence nets.

In Section 4 , we show an adjunction $\left\langle\left({ }_{-}\right)^{+}, \mathcal{u}[-]\right\rangle:$ DecOcc $\rightarrow$ PTNets whose right adjoint $\mathcal{u}[-]$ gives the unfoldings of PT nets. This adjunction restricts to Winskel's coreflection from Occ to Safe as shown by the commutative diagrams:
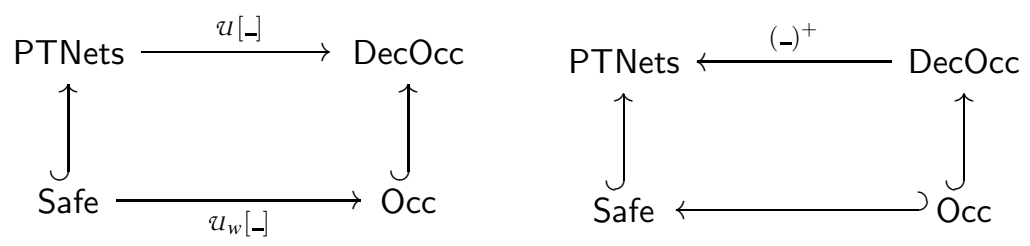

i.e., the left and the right adjoint, when restricted respectively to Safe and Occ, coincide with the corresponding adjoints of Winskel's coreflection. We also consider the full subcategory SWNets of PTNets consisting of those nets whose initial markings and whose post-sets are sets. Since weights are allowed only on the arcs from places to transitions, we refer to them as semiweighted nets. Concerning them, we show that when $\mathcal{u}[$ ] $]$ is restricted to SWNets the adjunction $\left\langle\left({ }_{-}\right)^{+}, \mathcal{u}[-]\right\rangle$ restrict to a coreflection $\langle\hookrightarrow, \mathcal{u}[-]\rangle:$ Occ $\rightarrow$ SWNets.

Then, in Section 5, we relate decorated occurrence nets to occurrence nets by showing an adjunction $\langle\mathcal{D}[-], \mathcal{F}[-]\rangle:$ Occ $\rightarrow$ DecOcc, where $\mathcal{F}[-]$ is the forgetful functor which forgets about 
families. Moreover, the diagram below commutes.

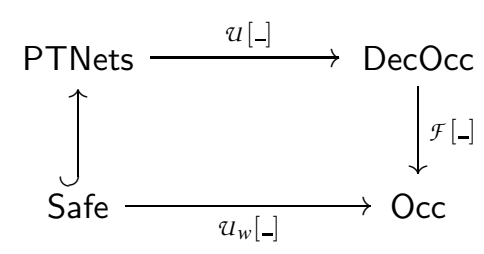

Therefore, we get the desired adjunction between Dom and PTNets as the composition of the chain of adjunctions

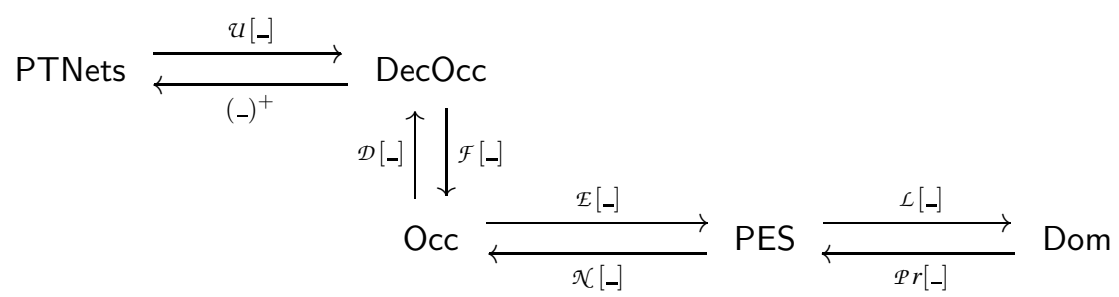

It follows from the commutative diagram (1) that, when PTNets is restricted to Safe, all the right adjoints in the above chain coincide with the corresponding functors defined by Winskel. Furthermore, because of the coreflection from Occ to SWNets, when $\mathcal{U}$ [-] is restricted to SWNets also $\mathcal{F} \mathcal{U}[$ [_], $\mathcal{E} \mathcal{F} \mathcal{U}[$ [-], and $\mathcal{L} \mathcal{E F} \mathcal{F}[$ [-] give rise to coreflections of, respectively, Occ, PES, and Dom in SWNets. In this sense, this work generalizes the work of Winskel and gives an abstract, truly concurrent semantics for PT nets. The existence of left adjoints for all the functors we consider guarantees the 'naturality' of this generalization. More precisely, as for safe nets, the adequate treatment of causal and true concurrency issues follows from the choice of event structures as denotational model, while the adjunctions guarantee that such a semantics is 'as good as possible', given the chosen categories. A further assessment of adequacy of the unfolding construction is provided by (Meseguer et al. 1996), where the present construction is proved to bear close relationships to the standard notion of processes for PT nets; more details about this are discussed in the concluding section. Another source of evidence is provided by the results about SWNets proving that for semiweighted nets, which can exhibit fairly more complicated causal behaviours than safe nets, the extension on Winskel's semantics is the smoothest possible one.

We remark that, of course, the semantics presented in this paper is one of the possible semantics for PT nets. In particular, it is characterized by the choice of prime event structures as 'denotations' of PT nets, i.e., by the choice of explaining the behaviour of PT nets in terms of the primitive notions of event, causality and conflict. Such a choice has the clear advantage of yielding a fully causal explanation of net computations, which is our declared aim. In fact, the unfolding functor associates to a PT net $N$ an occurrence net whose transitions represent exactly the instances of the transitions of $N$ in all the possible causal contexts and which can therefore account for concurrent multiple instances of the same element of $N$, that is for autoconcurrency. Of course, by its very purpose, the unfolding will then contain several instances of the same transition, including both concurrent pairs accounting for autoconcurrency and conflicting pairs arising from incompatible causal contexts. Clearly, this may be a disadvantage in 
some applications. These pros and cons are clearly sides of the same coin, and are both due to the 'primitiveness' of prime event structures.

This paper is a full and extend version of (Meseguer et al. 1992). With respect to that extended abstract version, the present paper provides the results concerning SWNets, further comments, and all the relevant proofs. In addition, here we also prove that the category PTNets has good structural properties by proving the existence of products and coproducts in PTNets and by providing a computational interpretation for them.

Acknowledgements. We cordially thank Narciso Martí-Oliet for his careful reading of the manuscript and his valuable suggestions for improving the exposition.

\section{Place/Transition Nets}

In this section we define the categories PTNets of Place/Transition (PT) nets, Safe of safe nets and Occ of occurrence nets.

We recall that a pointed set is a pair $(S, s)$ where $S$ is a set and $s \in S$ is the pointed element. Morphisms of pointed sets are functions that preserve the pointed elements. Looking at the pointed element as the undefined value, pointed sets provide a convenient way to treat partiality. More precisely, the category of sets and partial functions and the category of pointed sets and pointed set morphisms are isomorphic.

Given a set $S$, we denote by $S^{\mathcal{M}}$ the set of multisets on $S$, i.e., the set of all functions from $S$ to the set of natural numbers $\omega$, and by $S^{\mathcal{M} \infty}$ the set of multisets with (possibly) infinite multiplicities, i.e., the functions from $S$ to $\omega_{\infty}=\omega \cup\{\infty\}$. We shall regard $S^{\mathcal{M}}$ also as a pointed set whose pointed element is the empty multiset, i.e., the function which always yields zero, that, in the following, we shall denote by 0 .

Notation. For $\mu \in S^{\mathcal{M}_{\infty}}$, we write $[[\mu]]$ to denote the support of $\mu$, i.e., the subset of $S$ consisting of those elements $s$ such that $\mu(s)>0$. A multiset $\mu \in S^{\mathcal{M} \infty}$ can be represented as a formal sum $\bigoplus_{s \in S} \mu(s) \cdot s$. In the sequel, we shall often denote $\mu \in S^{\mathcal{M} \infty}$ by $\bigoplus_{i \in I} \eta_{i} s_{i}$ where $\left.\left\{s_{i} \mid i \in I\right\}=[\mu]\right]$ and $\eta_{i}=\mu\left(s_{i}\right)$, i.e., as a sum whose summands are all nonzero. In case of multisets in $S^{\mathcal{M}}$, instead of $\eta_{i}$, we shall use $n_{i}, m_{i}, \ldots$, the standard variables for natural numbers. Moreover, given $S^{\prime} \subseteq S$, we will write $\bigoplus S^{\prime}$ for $\bigoplus_{s \in S^{\prime}} 1 \cdot s=\bigoplus_{s \in S^{\prime}} s$.

Given an arbitrary indexing set $I$ and $\left\{\eta_{i} \in \omega_{\infty} \mid i \in I\right\}$, we define $\Sigma_{i \in I} \eta_{i}$ to be the usual sum in $\omega$ if only finitely many $\eta_{i}$ are nonzero and $\infty$ otherwise. Then, we can give meaning to linear combinations of multisets, i.e., multisets of multisets, by defining

$$
\bigoplus_{\mu \in S^{\mathcal{M} \infty}} \eta_{\mu} \cdot \mu=\bigoplus_{\mu \in S^{\mathcal{M}_{\infty}}} \eta_{\mu} \cdot\left(\bigoplus_{s \in S} \mu(s) \cdot s\right)=\bigoplus_{s \in S}\left(\sum_{\mu \in S^{\mathcal{M}}} \eta_{\mu} \mu(s)\right) \cdot s .
$$

As usual, we use the infix notation $u \oplus v$ when the above combination involves only the multisets $u$ and $v$ with multiplicities one.

$\mathrm{A}(-)^{\mathcal{M}_{\infty}}$-homomorphism from $S_{0}^{\mathcal{M} \infty}$ to $S_{1}^{\mathcal{M} \infty}$ is a function $g: S_{0}^{\mathcal{M} \infty} \rightarrow S_{1}^{\mathcal{M} \infty}$ such that

$$
g(\mu)=\bigoplus_{s \in S_{0}} \mu(s) \cdot g(s)
$$

Remark. (- $)^{\mathcal{M}_{\infty}}$ extends to an endofunctor on the category of sets and, as such, it defines a commutative monad, whose multiplication is the operation of linear combination of multisets described above, and 
whose unit maps $s \in S$ to $1 \cdot s \in S^{\mathcal{M}_{\infty}}$. In these terms, $S^{\mathcal{M}_{\infty}}$ is the free ()$^{\mathcal{M}_{\infty}}$-algebra on $S$ and a $\left({ }_{-}\right)^{\mathcal{M}_{\infty}}$ homomorphism is a morphism of ()$^{\mathcal{M}_{\infty}}$-algebras.

Definition 1.1. (PT Nets) A $P T$ net is a structure $N=\left(\partial_{N}^{0}, \partial_{N}^{1}:\left(T_{N}, 0\right) \rightarrow S_{N}^{\mathcal{M}}, u_{N}\right)$ where $S_{N}$ is a set whose elements are called places; $T_{N}$ is a pointed set whose elements are called transitions; $\partial_{N}^{0}, \partial_{N}^{1}$ are pointed set morphisms giving, respectively, sources and the targets of transitions; and $u_{N} \in S_{N}^{\mathcal{M}}$ is the initial marking. Moreover, we assume the standard constraint that if $\partial_{N}^{0}(t)=0$ then $t=0$.

A morphism of PT nets from $N_{0}$ to $N_{1}$ consists of a pair $\langle f, g\rangle$ such that:

(i) $\quad f: T_{N_{0}} \rightarrow T_{N_{1}}$ is a pointed set morphism;

(ii) $g: S_{N_{0}}^{\mathcal{M}_{\infty}} \rightarrow S_{N_{1}}^{\mathcal{M} \infty}$ is a $(-)^{\mathcal{M} \infty}$-homomorphism;

(iii) $g\left(u_{N_{0}}\right)=u_{N_{1}}$, i.e., $g$ respects the initial marking;

(iv) $\partial_{N_{1}}^{0} \circ f=g \circ \partial_{N_{0}}^{0}$, and $\partial_{N_{1}}^{1} \circ f=g \circ \partial_{N_{0}}^{1}$, i.e., $f$ respects source and target;

(v) $\forall a, b \in\left[\left[u_{N_{1}}\right]\right]$ and $\forall a, b \in\left[\left[\partial_{N_{0}}^{1}(t)\right]\right], t \in T_{N_{0}}$, if $[[g(a)]] \cap[[g(b)]] \neq \varnothing$, then $a=b$, i.e., $g$ acts 'disjointly' on places belonging to the initial marking or to the postset of the same transition.

This, with the obvious componentwise composition of morphisms, defines the category PTNets.

A PT net is thus a graph whose arcs are the transitions and whose nodes are the multisets on the set of places, i.e., markings of the net. As usual, transitions have pre- and post-sets, i.e., sources and targets, in which each place has only finitely many tokens, i.e., finite multiplicity. The same applies to the initial marking. Consistency with the use of zero transitions to treat partial maps is provided by the fact that $\partial_{N}^{0}$ and $\partial_{N}^{1}$ are pointed set morphisms, i.e., they assign empty pre- and post-sets to zero transitions. Moreover, these are the only transitions which can have empty pre-sets. This limitation is needed in any unfolding semantics since transitions with empty pre-sets are highly degenerated; in particular, any number of parallel copies of them can fire at any marking. Observe that PTNets contains also the empty net, i.e., in our setting the net with empty set of places and having the unique transition 0 . This is an interesting net, since it is the terminal object in the category. The initial object of PTNets is the net consisting of a unique place $s$, of no transitions, and whose initial marking is $1 \cdot s$.

Morphisms of PT nets are graph morphisms in the precise sense of respecting source and target of transitions, i.e., they make the two diagrams below commute.
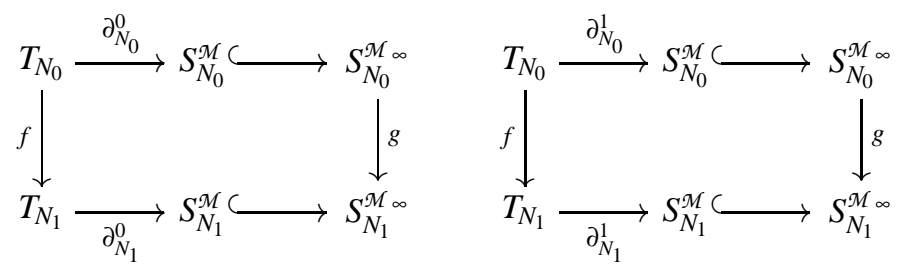

Moreover, they respect the pointed set structure of transitions, i.e., they are consistent with our use of 'zero' transitions, the $\left({ }_{-}\right)^{\mathcal{M} \infty}$-algebraic structures of states, and the initial markings.

The last condition in the definition means that morphisms are not allowed to map two different places in the initial marking or in the post-set of the same transition to two multisets having a place in common. This is pictorially described in the figure below, where dashed arrows represent the forbidden morphisms. We use the standard graphical representation of nets in which circles 
are places, boxes are transitions, the initial marking is given by the number of 'tokens' in the places, and sources and targets are directed arcs whose weights represent multiplicities. Unitary weights are omitted.
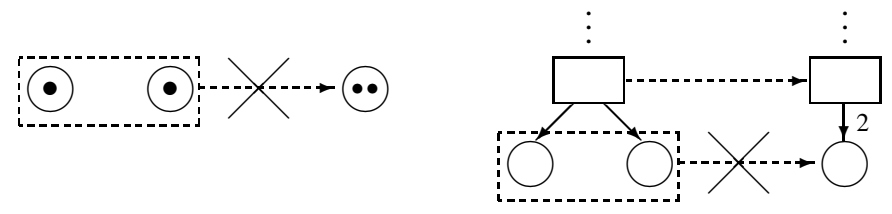

This requirement strengthen conditions (iii) and (iv), implying that multiplicities in the initial marking and in post-sets are 'reflected' by morphisms. It can be reformulated by saying that for all places $b \in\left[\left[u_{N_{1}}\right]\right]$, there exists exacly one $a \in\left[\left[u_{N_{0}}\right]\right]$ such that $b \in[[g(a)]]$ and, similarly, for all places $b \in\left[\left[\partial_{N_{1}}^{1}(f(t))\right]\right]$, for $t \in T_{N_{0}}$, there exists exactly one $a \in\left[\left[\partial_{N_{0}}^{1}(t)\right]\right.$ such that $b \in[[g(a)]]$. It is worth remarking that $(v)$ is a 'local' condition: it constrains the action of morphisms only on places belonging to the initial marking or to the same post-set. Such a condition will play an important role in establishing the adjunction between PTNets and DecOcc and, therefore, between PTNets and the other categories we consider. In fact, it is crucial for showing the (uniqueness condition in the definition of) universality of the counit of the adjunction. Moreover, it is exactly the part of this condition concerning the places in the initial marking which makes coproducts exist in PTNets.

Remark. Our choice of morphisms for PTNets is very close to those for the general categories of marked nets considered in (Winskel 1986; Winskel 1987; Meseguer and Montanari 1990) and in related works. Actually, the only difference with (Winskel 1986; Winskel 1987) is condition (v) in Definition 1.1.

Notation. Since in the rest of the paper we will often state and check conditions on both $\partial_{N}^{0}$ and $\partial_{N}^{1}$, we will use $\partial_{N}^{i}$ ranging over them. Moreover, in order to simplify notation, we shall sometimes use a single letter to denote a morphism $\langle f, g\rangle$. In these cases, the type of the argument will identify which component we are referring to. A ()$^{\mathcal{M}_{\infty}}$-homomorphism $g: S_{N_{0}}^{\mathcal{M} \infty} \rightarrow S_{N_{1}}^{\mathcal{M}_{\infty}}$, which constitutes the place component of a morphism $\langle f, g\rangle$, is completely defined by its behaviour on $S_{N_{0}}$, the generators of $S_{N_{0}}^{\mathcal{M}}$. Therefore, we will often define morphisms between nets by giving their transition components and a map $g: S_{N_{0}} \rightarrow S_{N_{1}}^{\mathcal{M}}$ for their place components: it is implicit that they have to be thought of as lifted to the correspondent $(-)^{\mathfrak{M}_{\infty}}$-homomorphisms.

Transitions are the basic units of computation in a PT net. A transition $t$ with $\partial_{N}^{0}(t)=u$ and $\partial_{N}^{1}(t)=v$-usually written $t: u \rightarrow v$-performs a computation consuming the tokens in $u$ and producing the tokens in $v$.

A finite number of transitions can be composed in parallel to form a step, which, therefore, is a finite multiset of transitions. We write $u[\alpha\rangle v$ to denote a step $\alpha$ with source $u$ and target $v$. The set $\mathcal{S}[N]$ of steps of $N$ is generated by the rules:

$$
\frac{t: u \rightarrow v \text { in } N \text { and } w \text { in } S^{\mathcal{M}} \infty}{(u \oplus w)[t\rangle(v \oplus w) \text { in } S[N]} \quad \frac{u[\alpha\rangle v \text { and } u^{\prime}[\beta\rangle v^{\prime} \text { in } S[N]}{\left(u \oplus u^{\prime}\right)[\alpha \oplus \beta\rangle\left(v \oplus v^{\prime}\right) \text { in } \mathcal{S}[N]}
$$

Observe that the pointed transition $0: 0 \rightarrow 0$ provides the empty step at any $u \in S^{\mathcal{M}_{\infty}}$.

A finite number of steps from the initial marking can be sequentially composed thus yielding 
a step sequence. The set of step sequences, denoted $\mathcal{S}[N]$, is given by the rule:

$$
\frac{u_{N}\left[\alpha_{0}\right\rangle v_{0}, \ldots, u_{n}\left[\alpha_{n}\right\rangle v_{n} \text { in } \mathcal{S}[N] \text { and } u_{i}=v_{i-1}, i=1, \ldots, n}{u_{N}\left[\alpha_{0}\right\rangle\left[\alpha_{1}\right\rangle \cdots\left[\alpha_{n}\right\rangle v_{n} \text { in } \mathcal{S} S[N]}
$$

The set $\mathcal{R}[N]$ of reachable markings of $N$ is the set of markings which are target of some step sequence, i.e.,

$$
\mathcal{R}[N]=\left\{v \mid \exists\left(u_{N}\left[\alpha_{0}\right\rangle \cdots\left[\alpha_{n}\right\rangle v\right) \text { in } \mathcal{S} \mathcal{S}[N]\right\} .
$$

Since step sequences are of finite length, and each step consists of finitely many transitions, from the conditions on $u_{N}, \partial_{N}^{0}$ and $\partial_{N}^{1}$ in Definition 1.1, it is easy to see that $\mathcal{R}[N] \subseteq S_{N}^{\mathscr{M}}$.

In the following we shall also consider the category of those PT nets whose initial markings and whose post-sets are sets, as opposed to multisets.

Definition 1.2. (SemiWeighted Nets) A PT net $N$ is semiweighted if $u_{N}$ is a set and, for all $t \in T_{N}, \partial_{N}^{1}(t)$ is a set. This defines the category SWNets as a full subcategory of PTNets.

It is interesting to notice that for the morphisms in SWNets condition $(v)$ is redundant, i.e., it is already implied by the structure of semiweighted nets.

Proposition 1.3. (Characterization of SemiWeighted Net Morphisms) Let $N_{0}$ and $N_{1}$ be semiweighted nets. A pair of $\langle f, g\rangle$, where $f: T_{N_{0}} \rightarrow T_{N_{1}}$ and $g: S_{N_{0}}^{\mathcal{M}} \rightarrow S_{N_{1}}^{\mathcal{M} \infty}$, is a morphism $N_{0} \rightarrow N_{1}$ in SWNets if and only if conditions (i), (ii), (iii) and (iv) of Definition 1.1 hold.

Proof. It is very easy to prove that condition $(v)$ follows from the others. In fact if there were $b$ in $\left[\left[u_{N_{1}}\right]\right]$ or in $\left[\left[\partial_{N_{1}}^{1}(f(t))\right]\right]$ and $a, a^{\prime}$ respectively in $\left[\left[u_{N_{0}}\right]\right]$ or in $\left[\left[\partial_{N_{0}}^{1}(t)\right]\right]$ such that $b \in[[g(a)]] \cap$ $\left[\left[g\left(a^{\prime}\right)\right]\right]$, by definition of morphism, it would be $g\left(u_{N_{0}}\right)(b)=u_{N_{1}}(b) \geq 2$ or $g\left(\partial_{N_{0}}^{1}(t)\right)(b)=$ $\partial_{N_{1}}^{1}(f(t))(b) \geq 2$. But this is impossible, since $N_{1}$ is a semiweighted net.

The important class of safe nets is obtained from SWNets by extending the required absence of multiplicities also to pre-sets and to all reachable markings.

Definition 1.4. (Safe Nets) A PT net $N$ is safe if

$$
\forall t \in T_{N}, \partial_{N}^{i}(t) \text { is a set, and } \forall v \in \mathcal{R}[N], v \text { is a set. }
$$

This defines the category Safe as a full subcategory of PTNets.

Since Safe is clearly a full subcategory of SWNets, Proposition 1.3 applies also to safe net morphisms. Moreover, the morphisms in Safe admit the following characterization in terms of their action on initial markings, pre- and post-sets.

Proposition 1.5. (Characterization of Safe Net Morphisms) Let $N_{0}$ and $N_{1}$ be safe nets. A pair $\langle f, g\rangle$ is a morphism $N_{0} \rightarrow N_{1}$ in Safe if and only if $f: T_{N_{0}} \rightarrow T_{N_{1}}$ is a morphism of pointed sets, $g: S_{N_{0}}^{\mathcal{M} \infty} \rightarrow S_{N_{1}}^{\mathcal{M} \infty}$ is a $\left(_{-}\right)^{\mathcal{M}}{ }^{\infty}$-homomorphism such that $\forall a \in\left[\left[u_{N_{0}}\right] \cup\left[\left[\partial_{N_{0}}^{0}(t)\right]\right] \cup\left[\partial_{N_{0}}^{1}(t)\right]\right.$, for $t \in T_{N_{0}}, g(a)$ is a set, and

(i) $\quad\left[\left[g\left(u_{N_{0}}\right)\right]\right] \subseteq\left[\left[u_{N_{1}}\right]\right]$ and $\forall b \in\left[\left[u_{N_{1}}\right]\right], \exists ! a \in\left[\left[u_{N_{0}}\right]\right]$ such that $b \in[[g(a)]$;

(ii) $\forall t \in T_{N_{0}},\left[\left[g\left(\partial_{N_{0}}^{i}(t)\right)\right]\right] \subseteq\left[\left[\partial_{N_{1}}^{i}(f(t))\right]\right]$ and

$$
\forall b \in\left[\left[\partial_{N_{1}}^{i}(f(t))\right]\right], \exists ! a \in\left[\left[\partial_{N_{0}}^{i}(t)\right]\right] \text { such that } b \in[[g(a)]] \text {. }
$$

Proof. $(\Rightarrow)$ Trivial.

$(\Leftarrow)$ Conditions $(i),(i i)$ and $(v)$ in the definition of PT net morphisms are already present. Points 
(i) and (ii) above imply that

$$
\left[\left[u_{N_{1}}\right] \rrbracket=\bigcup\left\{[g(a)] \mid a \in\left[\llbracket u_{N_{0}} \rrbracket\right\} \text { and }\left[\partial_{N_{1}}^{i}(f(t))\right]=\bigcup\{\llbracket g(a)] \mid a \in\left[\partial_{N_{0}}^{i}(t)\right]\right\}\right. \text {. }
$$

Now, since $\bigoplus\left[g(a) \rrbracket=g(a)\right.$ and all the $\llbracket g(a) \rrbracket$ in the unions are disjoint, we obtain $g\left(u_{N_{0}}\right)=u_{N_{1}}$ and $g\left(\partial_{N_{0}}^{i}(t)\right)=\partial_{N_{1}}^{i}(f(t))$.

Corollary 1.6. (Correspondence with Winskel's Safe Nets) Winskel's category of safe nets (Winskel 1986), called Net, is a full subcategory of Safe.

Proof. The conditions given in the above proposition are a characterization of morphisms in Net (Winskel 1986, Proposition 3.1.9), while the objects in Safe strictly contain the objects in Net. In fact, the objects of Net are the objects of Safe with sets of places, initial markings and post-sets which are non-empty, and without isolated places — places belonging neither to the initial marking nor to the pre- or post-set of any transition.

Another important class of nets is that of occurrence nets. They are safe nets which a nicely stratified structure whose minimal elements constitute the initial marking.

Notation. For $a \in S_{N}$, we use ${ }^{\bullet} a$ to indicate the set $\left\{t \in T_{N} \mid a \in\left[\partial_{N}^{1}(t)\right]\right\}$. Dually, $a^{\bullet}$ is $\left\{t \in T_{N} \mid a \in\right.$ $\left.\left[\llbracket \partial_{N}^{0}(t)\right]\right\}$. These notations are extended in the obvious way to sets of places.

Definition 1.7. (Occurrence Nets) An occurrence net is a safe net $\Theta$ such that

(i) $\left.a \in\left[u_{\Theta}\right]\right]$ if and only if ${ }^{*} a=\varnothing$;

(ii) $\forall a \in S_{\Theta},\left|\bullet^{\bullet} a\right| \leq 1$, where $\mid$-| gives the cardinality of sets;

(iii) $\prec$ is irreflexive, where $\prec$ is the transitive closure of the relation

$$
\prec^{1}=\left\{(a, t) \mid a \in S_{\Theta}, t \in T_{\Theta}, t \in a^{\bullet}\right\} \cup\left\{(t, a) \mid a \in S_{\Theta}, t \in T_{\Theta}, t \in \bullet^{\bullet} a\right\} ;
$$

moreover, $\forall t \in T_{\Theta},\left\{t^{\prime} \in T_{\Theta} \mid t^{\prime} \prec t\right\}$ is finite;

(iv) the binary 'conflict' relation \# on $T_{\Theta} \cup S_{\Theta}$ is irreflexive, where

$$
\begin{gathered}
\left.\forall t_{1}, t_{2} \in T_{\Theta}, t_{1} \#_{m} t_{2} \Leftrightarrow\left[\partial_{\Theta}^{0}\left(t_{1}\right)\right]\right] \cap\left[\llbracket \partial_{\Theta}^{0}\left(t_{2}\right)\right] \neq \varnothing \text { and } t_{1} \neq t_{2}, \\
\forall x, y \in T_{\Theta} \cup S_{\Theta}, x \# y \Leftrightarrow \exists t_{1}, t_{2} \in T_{\Theta}: t_{1} \#_{m} t_{2} \text { and } t_{1} \preceq x \text { and } t_{2} \preceq y,
\end{gathered}
$$

where $\preceq$ is the reflexive closure of $\prec$.

This defines the category Occ as a full subcategory of Safe.

From Definition 1.7 and Corollary 1.6, it is immediate to see that Winskel's category of occurrence nets (Winskel 1986), say Occw, is a full subcategory of Occ. However, since all the results in (Winskel 1986) easily extend to Safe and Occ, in the following we will ignore any difference between Safe and Net and between Occ and Occw.

\section{Composition of PT Nets}

Products and coproducts are important constructions for nets, and generally in categories of models for concurrency, due to their natural role, respectively, in the operations of parallel and nondeterministic composition (Winskel 1987). In this section, we show that the category PTNets has both products and coproducts and, studying the relationships between the computations of the composed nets and those of the original nets, we clarify in what sense products and coproducts are related to the operations of parallel and non-deterministic composition. 
While in the categories of safe nets and of occurrence nets products and coproducts exist (Winskel 1987), the category of PT nets with initial markings introduced in (Winskel 1987) has products but does not have coproducts. In (Meseguer and Montanari 1990), it is shown that coproducts exist in the full subcategory of PT nets whose initial markings are sets rather than multisets. However, due to the additional condition $(v)$ in Definition 1.1, we can prove the existence of coproducts of any pair of objects in PTNets.

Definition 2.1. (Product of Nets) Given the PT nets $N_{0}$ and $N_{1}$, we define

$$
N_{0} \times N_{1}=\left(\partial_{N_{0}}^{0} \times \partial_{N_{1}}^{0}, \partial_{N_{0}}^{1} \times \partial_{N_{1}}^{1}:\left(T_{N_{0}} \times T_{N_{1}},(0,0)\right) \rightarrow S_{N_{0}}^{\mathcal{M}} \times S_{N_{1}}^{\mathcal{M}},\left(u_{N_{0}}, u_{N_{1}}\right)\right) .
$$

Since $S_{N_{0}}^{\mathcal{M}} \times S_{N_{1}}^{\mathcal{M}} \cong\left(S_{N_{0}}+S_{N_{1}}\right)^{\mathcal{M}}$, and $S_{N_{0}}^{\mathcal{M}} \times S_{N_{1}}^{\mathcal{M}} \cong\left(S_{N_{0}}+S_{N_{1}}\right)^{\mathcal{M} \infty}$, where + is the disjoint union of sets, we have that $N_{0} \times N_{1}$ is indeed a net with places $S_{N_{0}}+S_{N_{1}}$. Example 2.2 illustrates the above definition in a simple case.

\section{Example 2.2.}
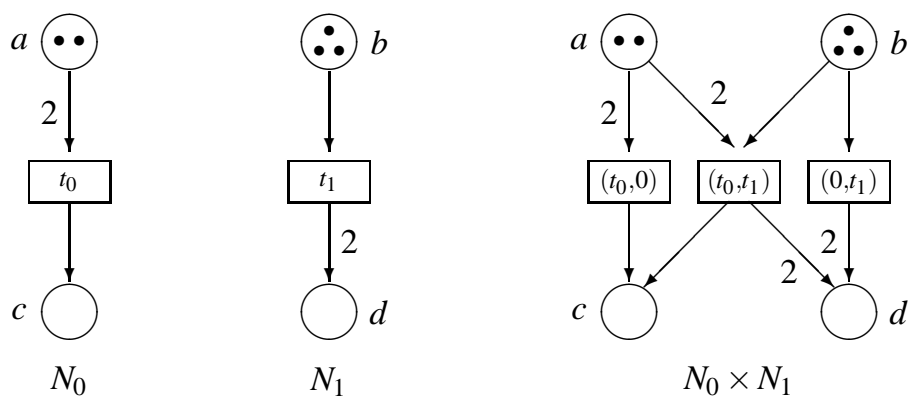

The PT nets $N_{0}$ and $N_{1}$ and their product $N_{0} \times N_{1}$

Now, consider the projections $\pi_{0}: N_{0} \times N_{1} \rightarrow N_{0}$ and $\pi_{1}: N_{0} \times N_{1} \rightarrow N_{1}$ defined by $\pi_{i}\left(\left(t_{0}, t_{1}\right)\right)=$ $t_{i}$ and $\pi_{i}\left(\left(u_{0}, u_{1}\right)\right)=u_{i}$. It is easy to see that they are morphisms in PTNets.

Proposition 2.3. (Product of Nets) $N_{0} \times N_{1}$, with projections $\pi_{0}$ and $\pi_{1}$, is the product of $N_{0}$ and $N_{1}$ in PTNets.

Proof. Observe that, given any PT net $N$ and two morphisms $h_{0}: N \rightarrow N_{0}$ and $h_{1}: N \rightarrow N_{1}$, the map $\left\langle h_{0}, h_{1}\right\rangle: N \rightarrow N_{0} \times N_{1}$ defined by

$$
\left\langle h_{0}, h_{1}\right\rangle(t)=\left(h_{0}(t), h_{1}(t)\right) \text { and }\left\langle h_{0}, h_{1}\right\rangle(u)=\left(h_{0}(u), h_{1}(u)\right)
$$

is a PT net morphism. Clearly, $\pi_{i} \circ\left\langle h_{0}, h_{1}\right\rangle=h_{i}$, and $\left\langle h_{0}, h_{1}\right\rangle$ is the unique morphism for which that happens.

The product of the nets $N_{0}$ and $N_{1}$ is their parallel composition with synchronization, in the precise sense that each step sequence of $N_{0} \times N_{1}$ is the parallel composition of a step sequence of $N_{0}$ and a step sequence of $N_{1}$, and viceversa. Since transitions of $N_{0} \times N_{1}$ are of the forms $\left(t_{0}, 0\right),\left(0, t_{1}\right)$ or $\left(t_{0}, t_{1}\right)$, for $t_{i} \in T_{N_{i}}, i=0,1$, the product models both asynchronous and synchronous interactions of $N_{0}$ and $N_{1}$, where transitions of the form $\left(t_{0}, 0\right)$ or $\left(0, t_{1}\right)$ correspond to either $N_{1}$ or $N_{0}$ staying idle, while transitions of the form $\left(t_{0}, t_{1}\right)$ correspond to steps in which both $N_{0}$ and $N_{1}$ proceed together, synchronizing to each other. This result, formally stated in the next proposition, coincides with those in (Winskel 1987; Meseguer and Montanari 1990). 
In the following, given a PT net morphism $h: N_{0} \rightarrow N_{1}$, we will denote by $h^{\oplus}$ the unique (_- $)^{\mathcal{M}_{\infty}}$ homomorphism from $T_{N_{0}}^{\mathcal{M} \infty}$ to $T_{N_{1}}^{\mathcal{M} \infty}$ generated by the transition component of $h$. Observe that, since such a component is a function, $h^{\oplus}$ maps finite multisets to finite multisets. In particular, $h^{\oplus}$ can be defined on such multisets simply by: $h^{\oplus}(t)=h(t)$ and $h^{\oplus}(\alpha \oplus \beta)=h^{\oplus}(\alpha) \oplus h^{\oplus}(\beta)$.

Proposition 2.4. (Product and Parallel Composition) The sequence $u_{N_{0} \times N_{1}}\left[\alpha_{0}\right\rangle \cdots\left[\alpha_{n}\right\rangle v$ belongs to $\mathcal{S S}\left[N_{0} \times N_{1}\right]$ if and only if the sequence $\pi_{i}\left(u_{N_{0} \times N_{1}}\right)\left[\pi_{i}^{\oplus}\left(\alpha_{0}\right)\right\rangle \cdots\left[\pi_{i}^{\oplus}\left(\alpha_{n}\right)\right\rangle \pi_{i}(v)$ belongs to $\mathcal{S S}\left[N_{i}\right]$, for $i=0,1$. Therefore, $v \in \mathcal{R}\left[N_{0} \times N_{1}\right]$ if and only if $\pi_{i}(v) \in \mathcal{R}\left[N_{i}\right]$ for $i=0,1$.

Proof. It suffices to show that $u[\alpha\rangle v$ is in $s\left[N_{0} \times N_{1}\right]$ if and only if $\pi_{i}(u)\left[\pi_{i}^{\oplus}(\alpha)\right\rangle \pi_{i}(v)$ is in $\mathcal{S}\left[N_{i}\right], i=0,1$.

Suppose $\alpha=\bigoplus_{j}\left(t_{0}^{j}, t_{1}^{j}\right)$. The 'only if' implication follows directly from the fact that $\pi_{0}$ and $\pi_{1}$ are PT net morphisms. In fact, by definition of $\mathcal{S}[N], u\left[\alpha>v\right.$ in $\mathcal{S}\left[N_{0} \times N_{1}\right]$ if and only if $u=$ $w \oplus \oplus_{j} u_{j}, v=w \oplus \oplus_{j} v_{j}$, and $\left(t_{0}^{j}, t_{1}^{j}\right): u_{j} \rightarrow v_{j}$ are transitions of $N_{0} \times N_{1}$. Thus, we have that $\pi_{i}\left(t_{0}^{j}, t_{1}^{j}\right): \pi_{i}\left(u_{j}\right) \rightarrow \pi_{i}\left(v_{j}\right)$ is a transition (possibly 0 ) of $N_{i}$. Therefore, for $i=0,1$,

$$
\left(\pi_{i}(w) \oplus \bigoplus_{j} \pi_{i}\left(u_{j}\right)\right)\left[\oplus_{j} \pi_{i}\left(t_{0}^{j}, t_{1}^{j}\right)\right\rangle\left(\pi_{i}(w) \oplus \bigoplus_{j} \pi_{i}\left(v_{j}\right)\right) \text { is in } \mathcal{S}\left[N_{i}\right],
$$

i.e., $\pi_{i}(u)\left[\pi_{i}^{\oplus}(\alpha)\right\rangle \pi_{i}(v)$ belongs to $\mathcal{S}\left[N_{i}\right]$.

In order to show the 'if' implication, observe that $t_{0}: \pi_{0}(u) \rightarrow \pi_{0}(v)$ in $N_{0}$ and $t_{1}: \pi_{1}(u) \rightarrow \pi_{1}(v)$ in $N_{1}$ imply $\left(t_{0}, t_{1}\right): u \rightarrow v$ in $N_{0} \times N_{1}$. Now assume that $\pi_{i}(u)\left[\pi_{i}^{\oplus}(\alpha)\right\rangle \pi_{i}(v)$ in $s\left[N_{i}\right], i=0,1$, $u=\left(u^{0}, u^{1}\right)$, and $v=\left(v^{0}, v^{1}\right)$. By definition of $s\left[N_{i}\right]$, we have $u^{i}=w^{i} \oplus \bigoplus_{j} u_{j}^{i}, v^{i}=w^{i} \oplus \bigoplus_{j} v_{j}^{i}$, and $\pi_{i}\left(t_{0}^{j}, t_{1}^{j}\right): u_{j}^{i} \rightarrow v_{j}^{i}$ in $N_{i}$. Now consider $u_{j}=\left(u_{j}^{0}, u_{j}^{1}\right), v_{j}=\left(v_{j}^{0}, v_{j}^{1}\right)$, and $w=\left(w^{0}, w^{1}\right)$. Clearly, we have $u=w \oplus \bigoplus_{j} u_{j}, v=w \oplus \bigoplus_{j} v_{j}$ and $\pi_{i}\left(t_{0}^{j}, t_{1}^{j}\right): \pi_{i}\left(u_{j}\right) \rightarrow \pi_{i}\left(v_{j}\right)$ in $N_{i}$. Therefore, $\left(t_{0}^{j}, t_{1}^{j}\right): u_{j} \rightarrow$ $v_{j}$ in $N_{i}$, and we have

$$
\left(w \oplus \bigoplus_{j} u_{j}\right)\left[\oplus_{j}\left(t_{0}^{j}, t_{1}^{j}\right)\right\rangle\left(w \oplus \bigoplus_{j} v_{j}\right) \text { in } \mathcal{S}\left[N_{0} \times N_{1}\right],
$$

i.e., $u[\alpha\rangle v$ in $S\left[N_{0} \times N_{1}\right]$.

Example 2.5. (Parallel Computations) Consider again the nets of Example 2.2.

The step $(2 a, 3 b)\left[\left(t_{0}, t_{1}\right)\right\rangle(c, 2 b \oplus 2 d)$ of $N_{0} \times N_{1}$ corresponds to the steps $2 a\left[t_{0}\right\rangle c$ of $N_{0}$ and $3 b\left[t_{1}\right\rangle 2 b \oplus 2 d$ of $N_{1}$, while $(2 a, 3 b)\left[\left(t_{0}, 0\right)\right\rangle\left[\left(0, t_{1}\right)\right\rangle(c, 2 b \oplus 2 d)$ corresponds to $2 a\left[t_{0}\right\rangle[0\rangle c$ and to $3 b[0\rangle\left[t_{1}\right\rangle 2 b \oplus 2 d$.

We now consider coproducts in PTNets. To this purpose, letting $\oplus$ stand for the coproduct in the category of multisets (with possibly infinite multiplicities) and (_- $)^{\mathcal{M}_{\infty}}$-homomorphisms, observe that $S_{0}^{\mathcal{M}_{\infty}} \oplus S_{1}^{\mathcal{M}_{\infty}} \cong\left(S_{0}+S_{1}\right)^{\mathcal{M}_{\infty}}$. Given the PT nets $N_{0}$ and $N_{1}$, let $u_{N_{0}}=\bigoplus_{i} n_{i} a_{i}$ and $u_{N_{1}}=\bigoplus_{j} m_{j} b_{j}$, and consider the set $S_{N_{0}+N_{1}}=\left(S_{N_{0}}-\llbracket\left[u_{N_{0}} \rrbracket\right)+\left(S_{N_{1}}-\left[\llbracket u_{N_{1}}\right]\right)+\left(\llbracket u_{N_{0}}\right]\right) \times\left[u_{N_{1}} \rrbracket\right)$ and the $(-)^{\mathcal{M}_{\infty}}$-homomorphisms $\alpha_{i}:\left(\left[u_{N_{i}} \rrbracket\right)^{\mathcal{M}_{\infty}} \rightarrow\left(\left[u_{N_{0}}\right]\right] \times\left[\left[u_{N_{1}}\right]\right)^{\mathscr{M}_{\infty}}, i=0,1\right.$, defined by

$$
\begin{aligned}
& \alpha_{0}\left(a_{i}\right)=\bigoplus_{j} \frac{\operatorname{lcm}\left(n_{i}, m_{j}\right)}{n_{i}}\left(a_{i}, b_{j}\right) \\
& \alpha_{1}\left(b_{j}\right)=\bigoplus_{i} \frac{\operatorname{lcm}\left(n_{i}, m_{j}\right)}{m_{j}}\left(a_{i}, b_{j}\right),
\end{aligned}
$$

where lcm denotes the least common multiple. For $i=0,1$, let $\gamma_{i}$ be $\alpha_{i} \oplus \beta_{i}: S_{N_{i}}^{\mathscr{M}} \rightarrow S^{\mathcal{M}_{\infty}}$ where $\beta_{i}$ is the injection of $\left(S_{N_{i}}-\llbracket u_{N_{i}} \rrbracket\right)^{\mathcal{M} \infty}$ in $\left(S_{N_{0}}-\llbracket\left[u_{N_{0}} \rrbracket+S_{N_{1}}-\llbracket\left[u_{N_{1}} \rrbracket\right)^{\mathcal{M} \infty}\right.\right.$, and let $\delta_{N_{j}}^{i}$ be $\gamma_{j} \circ$ $\partial_{N_{j}}^{i}:\left(T_{N_{j}}, 0\right) \rightarrow S_{N_{0}+N_{1}}^{\mathcal{M}}$, for $i, j=0,1$. 
Definition 2.6. (Coproduct of Nets) For $N_{0}$ and $N_{1}$ PT nets, define

$$
N_{0}+N_{1}=\left(\left[\delta_{N_{0}}^{0}, \delta_{N_{1}}^{0}\right],\left[\delta_{N_{0}}^{1}, \delta_{N_{1}}^{1}\right]:(T, 0) \rightarrow S_{N_{0}+N_{1}}^{\mathcal{M}}, \gamma_{0}\left(u_{N_{0}}\right)=\gamma_{1}\left(u_{N_{1}}\right)\right),
$$

where $(T, 0)$ is the coproduct of pointed sets $\left(T_{N_{0}}, 0\right)$ and $\left(T_{N_{1}}, 0\right)$, i.e., the quotient of their disjoint union obtained by identifying the two pointed elements, and $\left[\delta_{N_{0}}^{i}, \delta_{N_{1}}^{i}\right]$ denotes the unique pointed set morphism induced from the coproduct $(T, 0)$ by $\delta_{N_{0}}^{i}$ and $\delta_{N_{1}}^{i}$.

\section{Example 2.7.}
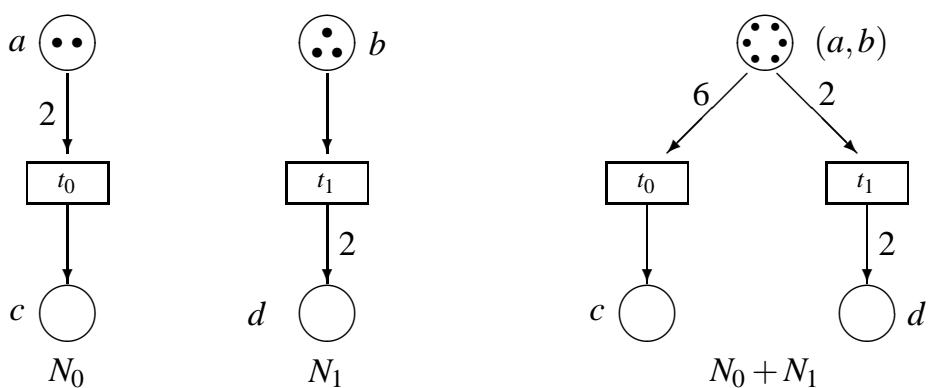

The PT nets $N_{0}$ and $N_{1}$ and their coproduct $N_{0}+N_{1}$

The injections $i n_{i}: N_{i} \rightarrow N_{0}+N_{1}, i=0,1$, are defined as

$$
i n_{i}=\left\langle\kappa_{i}, \gamma_{i}\right\rangle
$$

where $\kappa_{i}$ is the injection of $\left(T_{N_{i}}, 0\right)$ in $(T, 0)$. It is immediate to see that the $i n_{i}$ are PT net morphisms.

Given a PT net $N$ and a pair of morphisms $h_{0}: N_{0} \rightarrow N$ and $h_{1}: N_{1} \rightarrow N$, let $\left[h_{0}, h_{1}\right]: N_{0}+N_{1} \rightarrow N$ be the map such that

$$
\begin{aligned}
{\left[h_{0}, h_{1}\right](t) } & =h_{i}\left(t^{\prime}\right) \quad \text { if } t=i n_{i}\left(t^{\prime}\right) \text { for } t^{\prime} \in T_{N_{i}} \\
{\left[h_{0}, h_{1}\right](c) } & =h_{i}\left(c^{\prime}\right) \quad \text { if } c=i n_{i}\left(c^{\prime}\right) \text { for } c^{\prime} \in S_{N_{i}}-\left[\left[u_{N_{i}}\right]\right] \\
{\left[h_{0}, h_{1}\right]\left(a_{i}, b_{j}\right) } & =\bigoplus\left\{\frac{n_{k}}{\operatorname{lcm}\left(n_{i}, m_{j}\right)} c_{k} \mid c_{k} \in\left[\left[h_{0}\left(a_{i}\right)\right]\right] \cap\left[\left[h_{1}\left(b_{j}\right)\right]\right]\right\}
\end{aligned}
$$

where $n_{k}$ is the coefficient of $c_{k}$ in $u_{N}$. To simplify the notation, in the proof of the following proposition we will denote $\left[\left[h_{0}\left(a_{i}\right)\right]\right] \cap\left[\left[h_{1}\left(b_{j}\right)\right]\right]$ by $\left[\left[\mathfrak{I}\left(a_{i}, b_{j}\right)\right]\right]$.

Proposition 2.8. (Coproduct of Nets) $N_{0}+N_{1}$, with injections $i n_{0}$ and $i n_{1}$, is the coproduct of $N_{0}$ and $N_{1}$ in PTNets.

Proof. We show that for any PT net $N$ and for any pair ot PT net morphisms $h_{0}: N_{0} \rightarrow N$, $h_{1}: N_{1} \rightarrow N,\left[h_{0}, h_{1}\right]$ is the unique morphism in PTNets such that $\left[h_{0}, h_{1}\right] \circ i n_{i}=h_{i}$. First we have to show that $\left[h_{0}, h_{1}\right]$ is well-defined, i.e., that $n_{k} / \operatorname{lcm}\left(n_{i}, m_{j}\right)$ is actually a natural number. If $c_{k} \in\left[\left[h_{0}\left(a_{i}\right)\right]\right]$ then $h_{0}\left(a_{i}\right)=r_{k} c_{k} \oplus u$ and so $h_{0}\left(n_{i} a_{i}\right)=n_{i} r_{k} c_{k} \oplus n_{i} u$. Thus, by definition of PT net morphisms, we know that $u_{N}\left(c_{k}\right)=n_{i} r_{k}$ and so it must be $n_{i} r_{k}=n_{k}$. In the same way, there exists $q_{k}$ such that $m_{j} q_{k}=n_{k}$. Therefore $n_{k}$ is divisible by $\operatorname{lcm}\left(n_{i}, m_{j}\right)$.

Now, observe that $\left[h_{0}, h_{1}\right] \circ i n_{i}=h_{i}$. This is clear for transitions and for places in $S_{N_{i}}-\left[\left[u_{N_{i}}\right]\right]$. So, 
consider $a_{i} \in\left[\left[u_{N_{0}}\right]\right.$. . We have

$$
\begin{aligned}
{\left[h_{0}, h_{1}\right]\left(i n_{0}\left(a_{i}\right)\right) } & =\left[h_{0}, h_{1}\right]\left(\oplus_{j} \frac{\operatorname{lcm}\left(n_{i}, m_{j}\right)}{n_{i}}\left(a_{i}, b_{j}\right)\right) \\
& =\oplus_{j} \frac{\operatorname{lcm}\left(n_{i}, m_{j}\right)}{n_{i}}\left[h_{0}, h_{1}\right]\left(\left(a_{i}, b_{j}\right)\right) \\
& =\bigoplus_{j} \frac{\operatorname{lcm}\left(n_{i}, m_{j}\right)}{n_{i}} \bigoplus\left\{\frac{n_{k}}{\operatorname{lcm}\left(n_{i}, m_{j}\right)} c_{k} \mid c_{k} \in\left[\mathfrak{I}\left(a_{i}, b_{j}\right)\right]\right\} \\
& =\bigoplus_{j}\left\{\frac{n_{k}}{n_{i}} c_{k} \mid c_{k} \in\left[\mathfrak{I}\left(a_{i}, b_{j}\right)\right]\right\} .
\end{aligned}
$$

Since for each $\left.c_{k} \in \llbracket h_{0}\left(a_{i}\right) \rrbracket\right]$ there exists a unique $b_{j}$ such that $c_{k} \in\left[h_{1}\left(b_{j}\right) \rrbracket\right.$, the last term is equal to

$$
\bigoplus\left\{\frac{n_{k}}{n_{i}} c_{k} \mid c_{k} \in\left[h_{0}\left(a_{i}\right)\right]\right\}=\bigoplus\left\{r_{k} c_{k} \mid h_{0}\left(a_{i}\right)=r_{k} c_{k} \oplus u^{\prime}\right\}=h_{0}\left(a_{i}\right) .
$$

The same argument goes through for $b_{j} \in\left[\left[u_{N_{1}}\right]\right]$.

To prove uniqueness, suppose that there exists $h$ such that $h \circ i n_{i}=h_{i}$. Clearly, $h=\left[h_{0}, h_{1}\right]$ on the transitions and on places in $S_{N_{0}}-\left[\left[u_{N_{0}}\right]\right]$ and in $S_{N_{1}}-\left[\left[u_{N_{1}}\right]\right.$. Therefore, in order to show that $h$ coincides with $\left[h_{0}, h_{1}\right]$ we need to show that it does so for $\left(a_{i}, b_{j}\right) \in\left[\left[u_{N_{0}}\right]\right] \times\left[\left[u_{N_{1}}\right]\right.$. Since $h\left(i n_{0}\left(a_{i}\right)\right)=h_{0}\left(a_{i}\right)$ and $h$ is a morphism, we have

$$
\begin{aligned}
\bigoplus_{j} \frac{\operatorname{lcm}\left(n_{i}, m_{j}\right)}{n_{i}} h\left(a_{i}, b_{j}\right)= & \oplus\left\{\frac{n_{k}}{n_{i}} c_{k} \mid c_{k} \in\left[h_{0}\left(a_{i}\right)\right]\right\} \\
= & \left.\bigoplus_{j}\left\{\frac{n_{k}}{n_{i}} c_{k} \mid c_{k} \in\left[\mathfrak{I}\left(a_{i}, b_{j}\right)\right]\right\}\right\} \\
= & \bigoplus_{j} \frac{\operatorname{lcm}\left(n_{i}, m_{j}\right)}{n_{i}} \\
& \cdot\left(\oplus\left\{\frac{n_{k}}{\operatorname{lcm}\left(n_{i}, m_{j}\right)} c_{k} \mid c_{k} \in\left[\mathfrak{I}\left(a_{i}, b_{j}\right)\right]\right\}\right) .
\end{aligned}
$$

In the same way, we obtain that

$$
\bigoplus_{i} \frac{\operatorname{lcm}\left(n_{i}, m_{j}\right)}{m_{j}} h\left(a_{i}, b_{j}\right)=\oplus_{i} \frac{\operatorname{lcm}\left(n_{i}, m_{j}\right)}{m_{j}} \oplus\left\{\frac{n_{k}}{\operatorname{lcm}\left(n_{i}, m_{j}\right)} c_{k} \mid c_{k} \in\left[\mathfrak{I}\left(a_{i}, b_{j}\right)\right]\right\} .
$$

Now fix $i$ and $j$. Since $\left.c_{k} \in \llbracket \mathfrak{I}\left(a_{i}, b_{j}\right)\right]$ for a unique pair $\left(a_{i}, b_{j}\right)$, the summands in the above equalities are all distinct except for $\bigoplus\left\{\frac{n_{k}}{\operatorname{lcm}\left(n_{i}, m_{j}\right)} c_{k} \mid c_{k} \in\left[\left\{\mathfrak{I}\left(a_{i}, b_{j}\right)\right]\right\}\right.$, which appears in both. Therefore it must be

$$
h\left(a_{i}, b_{j}\right)=\bigoplus\left\{\frac{n_{k}}{\operatorname{lcm}\left(n_{i}, m_{j}\right)} c_{k} \mid c_{k} \in\left[\left[\mathfrak{I}\left(a_{i}, b_{j}\right)\right]\right\}\right\} \text { which is }\left[h_{0}, h_{1}\right]\left(a_{i}, b_{j}\right) .
$$

The last thing we have left to show is that $\left[h_{0}, h_{1}\right]$ is a morphism in PTNets. But now this task is trivial and is therefore omitted.

The coproduct of $N_{0}$ and $N_{1}$ is their non-deterministic composition in the sense that the two nets are put side by side to compete for common resources (tokens). Differently from the CCS non-deterministic operator (Milner 1989), the result of such a composition cannot be seen simply as the system which performs an initial choice between passing the control to $N_{0}$ or to $N_{1}$ and discards the net which has not been chosen. Nevertheless, we think that it gives the right notion of non-deterministic composition of PT nets. In fact, since a resource can be consumed and produced several times during a single computation, it is possible that the composed net returns several times to a state in which common resources are present and the two nets compete for 
them. Clearly, there is no reason why the outcome of such competitions should always favor the same net.

Example 2.9.
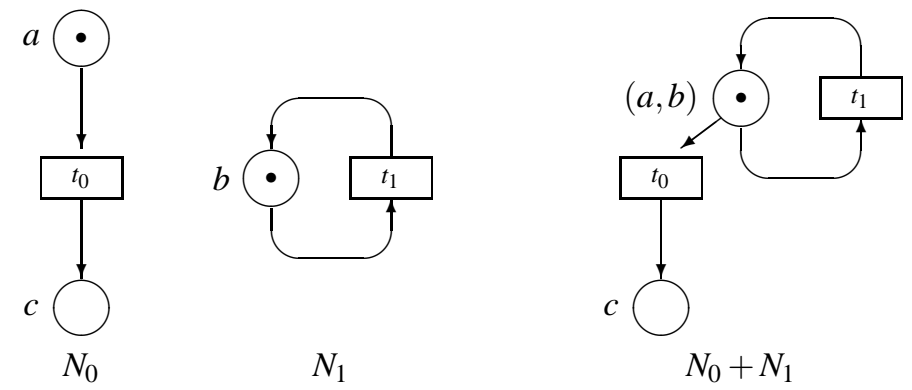

The initial marking $(a, b)$ of $N_{0}+N_{1}$ is a state in which a resource is non-deterministically assigned either to $t_{0}$ or to $t_{1}$. This state is reached again and again, and each time the choice is repeated.

It is easy to think of pratical situations which can be successfully described by this kind composition. For instance, in the example above, $t_{0}$ may be a process locking, accessing, and unlocking a file (whose local name is $b$ ), while $t_{0}$ may be a process trying to lock the same file (with local name $a$, though) in order to remove it. When $t_{0}$ succeeds, it makes the file not available anymore to $t_{1}$.

The kind of behaviour illustrated above is characteristic of the coproduct in many categories of models which admit cyclic behaviours, like, for instance, transition systems. In addition to that, since the resources are present in multiple instances (multiple tokens in a place), while the computations of $N_{0}$ and $N_{1}$ are also computations of $N_{0}+N_{1}$, they are not the only computations that the coproduct net can perform: the non-deterministic interaction between $N_{0}$ and $N_{1}$ gives rise to joint computations which are not purely injections of computations from one of the original nets. In other words, since providing $N_{0}$ with the resources it needs does not necessarily consume all the available instances of such resources, it is possible that $N_{1}$ can also have, at the same time, other instances of the same resources. This is shown by the following example.

\section{Example 2.10. (Non-Deterministic Computations)}
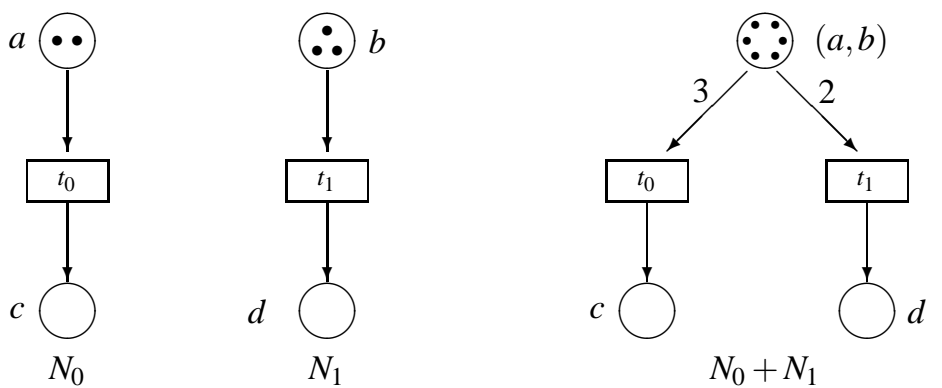

The steps $6(a, b)\left[2 t_{0}\right\rangle 2 c$ and $6(a, b)\left[3 t_{1}\right\rangle 3 d$ of $N_{0}+N_{1}$ correspond to the step $2 a\left[2 t_{0}\right\rangle 2 c$ of $N_{0}$ and to the step $3 b\left[3 t_{1}\right\rangle 3 d$ of $N_{1}$, respectively. 
The step $6(a, b)\left[t_{0} \oplus t_{1}\right\rangle c \oplus d$ is a computation of $N_{0}+N_{1}$ which is not the image of a computation in one of the original nets.

Another practical example modelled by this construction may be the following. Suppose that $2 a$ and $3 b$ both model a memory of $6 \mathrm{Mb}$ which $t_{0}$ sees as partitioned in two blocks by $3 \mathrm{Mb}$ and $t_{1}$ as partitioned in three blocks by $2 \mathrm{Mb}$. Then, a firing of $t_{0}$ (of $t_{1}$ ) represents the consumption of $2 \mathrm{Mb}$ (of $3 \mathrm{Mb}$ ) of memory assigned to $t_{0}$ (to $t_{1}$ ). When the processes are put side by side to compete for the available resources, i.e., $6 \mathrm{MB}$ of memory, the possible outcomes are exactly described by the net $N_{0}+N_{1}$ above, where the tokens now represent a partition of the memory in six blocks by $1 \mathrm{Mb}$.

As anticipated above, all the computations which either $N_{0}$ or $N_{1}$ can perform are computations which $N_{0}+N_{1}$ can perform; viceversa, all the computations of $N_{0}+N_{1}$ consisting of markings and steps from $N_{i}$ are actually computations of $N_{i}$. This is stated in the next proposition, whose proof simply follows from the fact that $i n_{0}$ and $i n_{1}$ are PT net morphisms and is, therefore, omitted.

Proposition 2.11. (Coproduct and Non-Deterministic Composition) The sequence of steps $i n_{i}\left(u_{N_{i}}\right)\left[i n_{i}^{\oplus}\left(\alpha_{0}\right)\right\rangle \cdots\left[i n_{i}^{\oplus}\left(\alpha_{n}\right)\right\rangle i n_{i}(v)$ belongs to $\mathcal{S} S\left[N_{0}+N_{1}\right]$ if and only if the sequence of steps $u_{N_{i}}\left[\alpha_{0}\right\rangle \cdots\left[\alpha_{n}\right\rangle v$ belongs to $\mathcal{S}\left[N_{i}\right]$.

In order to strengthen the intuition about the coproduct construction, it is worth recalling that in the case of safe nets all the resources are present in a unique copy. This fact can be thought of as forcing a choice between the two nets in the assignment of resources. Therefore, for safe nets, the computations of $N_{0}+N_{1}$ are alternating sequences of computations of the original nets, i.e., each step is either a step of $N_{0}$ or a step of $N_{1}$. This is stated in the next proposition, which is a rephrasing in the present context of (Winskel 1987, Theorem 5.11, pg. 219) and whose proof is, therefore, omitted.

Proposition 2.12. (Coproduct and Safe Nets) Let $N_{0}$ and $N_{1}$ be safe nets. Then $u[\alpha\rangle v$ is in $\mathcal{S}\left[N_{0}+N_{1}\right]$, for $u \in \mathcal{R}\left[N_{0}+N_{1}\right]$, if and only if there exist $i \in\{0,1\}, u^{\prime} \in \mathcal{R}\left[N_{i}\right]$, and $u^{\prime}\left[\alpha^{\prime}\right\rangle v^{\prime}$ in $\mathcal{S}\left[N_{i}\right]$ such that $i n_{i}\left(u^{\prime}\right)=u, \operatorname{in}_{i}\left(v^{\prime}\right)=v, \operatorname{in}_{i}^{\oplus}\left(\alpha^{\prime}\right)=\alpha$.

It follows that all the step sequences of $N_{0}+N_{1}$ are of the form

$$
i n_{i_{0}}\left(u_{N_{i_{0}}}\right)\left[i n_{i_{0}}^{\oplus}\left(\alpha_{0}\right)\right\rangle\left[i n_{i_{1}}^{\oplus}\left(\alpha_{1}\right)\right\rangle \cdots\left[i n_{i_{(k-1)}}^{\oplus}\left(\alpha_{(k-1)}\right)\right\rangle\left[i n_{i_{k}}^{\oplus}\left(\alpha_{k}\right)\right\rangle i n_{i_{k}}\left(v_{i_{k}}\right)
$$

where $i_{1}, \ldots, i_{k} \in\{0,1\}, v_{i_{k}} \in \mathcal{R}\left[N_{i_{k}}\right]$, and $\alpha_{j}$ is a step of $N_{i_{j}}, j=1, \ldots, k$.

Therefore, $u \in \mathcal{R}\left[N_{0}+N_{1}\right]$ if and only if $u=i n_{i}\left(u_{i}\right)$ for $u_{i} \in \mathcal{R}\left[N_{i}\right]$ and $i \in\{0,1\}$.

It is interesting to observe how in this case the standard coproduct construction actually implements a sophisticated mechanism of distributed choice. Consider two safe nets $N_{0}$ and $N_{1}$ whose initial markings are respectively $a_{1} \oplus \cdots \oplus a_{n}$ and $b_{1} \oplus \cdots \oplus b_{m}$. Then, the initial marking 
of $N_{0}+N_{1}$ can be thought of as an $n \times m$ matrix whose $(i, j)$ th entry represents the token $\left(a_{i}, b_{j}\right)$.

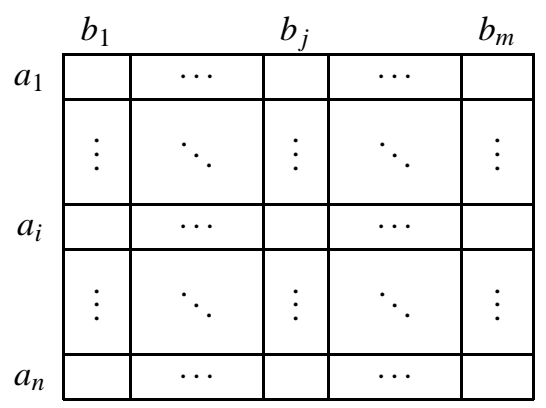

It follows from the definition of $\partial_{N_{0}+N_{1}}^{0}$ that if $a_{i}$ is in the pre-set of a transition $t_{0}$ in $N_{0}$, then the pre-set of $t_{0}$ in $N_{0}+N_{1}$ contains $\left(a_{i}, b_{1}\right) \oplus \cdots \oplus\left(a_{i}, b_{m}\right)$, i.e., a whole row of the matrix. Now, since a transition $t_{0}$ of $N_{0}$ enabled at $u_{N_{0}}$ requires at least one of the tokens in $u_{N_{0}}$ in order to fire, say $a_{i}$, the firing of $t_{0}$ in $N_{0}+N_{1}$ will consume all the tokens in the $i$ th row of the matrix. It follows that no transition of $N_{1}$ can be enabled, since for any $j=1, \ldots, m$, the token $\left(a_{i}, b_{j}\right)$ is missing. Therefore, the firing of $t_{0}$ prevents any transition of $N_{1}$ from firing until the possibly cyclic behaviour of $N_{0}+N_{1}$ eventually generates again the tokens in $u_{N_{0}+N_{1}}$.

We conclude this discussion about coproducts considering the case of occurrence nets. Since cyclic behaviours are not possible in occurrence nets, the coproduct net, after having performed the first step, cannot reach anymore a state in which common resources are available. In this case, therefore, the coproduct net can be seen as the system which performs an initial choice between the original nets - by assigning to one of them the resources it needs - and forgets about the other. This is formally stated in the following proposition.

Proposition 2.13. (Coproduct and Occurrence Nets) Let $\Theta_{0}$ and $\Theta_{1}$ be occurrence nets. Then, all the step sequences of $\Theta_{0}+\Theta_{1}$ from the initial marking are of the form

$$
\operatorname{in}_{i}\left(u_{\Theta_{i}}\right)\left[i n_{i}^{\oplus}\left(\alpha_{0}\right)\right\rangle \cdots\left[i n_{i}^{\oplus}\left(\alpha_{k}\right)\right\rangle i n_{i}\left(v_{i}\right),
$$

where $i \in\{0,1\}, v_{i} \in \mathcal{R}\left[\Theta_{i}\right]$, and $\alpha_{j}$ is a step of $\Theta_{i}, j=1, \ldots k$.

We conclude this section with some remarks about the relationships between products and coproducts in the other categories of nets introduced in Section 1.

It is easy to see that products and coproducts of semiweighted and safe nets viewed as objects in PTNets are again, respectively, semiweighted and safe nets. Therefore, products and coproducts exist in SWNets and Safe and they are given by the corresponding constructions we defined for PTNets. The same applies to coproducts in Occ.

However, the product of two occurrence nets in PTNets is not necessarily an occurrence net. This can be seen by looking back at Example 2.2, which shows that condition (ii) in Definition 1.7 of occurrence nets is not preserved by the product construction. Nevertheless, products exist in Occ. In particular, the result that the product of two occurrence nets is (isomorphic to) the unfolding of their product in as safe nets (Winskel 1987) can be immediately extended to our setting by considering that the unfolding of PT nets defined here coincides on safe nets with Winskel's (see the following Theorem 4.8). 


\section{Decorated Occurrence Nets}

In this section, we introduce DecOcc, the category of decorated occurrence nets, a type of occurrence nets in which places are grouped into families. They allow a convenient treatment of multiplicity issues in the unfolding of PT nets. We shall use the following notational conventions:

$$
\begin{aligned}
{[n, m] } & \text { for the segment }\{n, \ldots, m\} \text { of } \omega ; \\
{[n] } & \text { for }[1, n] ; \\
{[k]_{i} } & \text { for the } i \text { th block of length } k \text { of } \omega-\{0\}, \text { i.e., }[i k]-[(i-1) k] .
\end{aligned}
$$

Definition 3.1. (Block Functions) We call a function $f:[n] \rightarrow[m]$ a block function if and only if $n=k m$ and $f\left([k]_{i}\right)=\{i\}$, for $i=1, \ldots, m$.

In other words, a block function from $[n]=[\mathrm{km}]$ to $[\mathrm{m}]$ is a function making the diagram

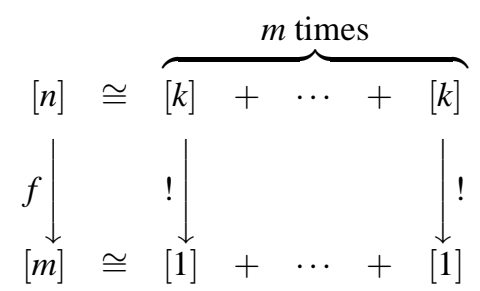

commute, where the upper isomorphism maps the segment $[k]_{i}$ to the $i$ th copy of $[k]$, and the lower maps $i$ to the $i$ th copy of [1].

The place component $g$ of a PT net morphism $\langle f, g\rangle: N_{0} \rightarrow N_{1}$ can be thought of as a multirelation (with possibly infinite multiplicities) between $S_{N_{0}}$ and $S_{N_{1}}$, namely the multirelation $g$ such that $a g \eta b$ if and only if $g(a)(b)=\eta$. Indeed, this is a (generalization of a) widely used formalization of net morphisms due to Winskel (Winskel 1984; Winskel 1987). In the case of morphisms between occurrence nets, since by definition such nets have no isolated places-i.e., places belonging neither to the initial marking nor to any pre- or post-set-as an immediate corollary to Proposition 1.5, we have that $g$ is a relation and that the inverse relation $g^{o p}$, defined by $b g^{o p} a$ if and only if $a g b$, restricts to (total) functions $g_{\varnothing}^{o p}:\left[\left[u_{N_{1}}\right]\right] \rightarrow\left[\left[u_{N_{0}}\right]\right]$ and $g_{\{t\}}^{o p}:\left[\left[\partial_{N_{1}}^{1}(f(t))\right]\right] \rightarrow\left[\left[\partial_{N_{0}}^{1}(t)\right]\right]$ for each $t \in T_{N_{0}}$. We will use these functions in the next definition.

Definition 3.2. (Decorated Occurrence Nets) A decorated occurrence net is an occurrence net $\Theta$ such that:

(i) $S_{\Theta}$ is of the form $\bigcup_{a \in A_{\Theta}}\{a\} \times\left[n_{a}\right]$, for some set $A_{\Theta}$, where the set $\{a\} \times\left[n_{a}\right]$ is called the family of $a$. We will use $a^{F}$ to denote the family of $a$ regarded as a multiset;

(ii) $\forall a \in A_{\Theta}, \forall x, y \in\{a\} \times\left[n_{a}\right], \bullet x=\bullet y$.

A morphism of decorated occurrence nets $\langle f, g\rangle: \Theta_{0} \rightarrow \Theta_{1}$ is a morphism of occurrence nets which respects families, i.e., for each $\left[\left[a^{F}\right]\right] \subseteq S_{\Theta_{0}}$, given $x=\bullet\left[\left[a^{F}\right]\right]$ - which is a singleton set or the empty set by (ii) above and the definition of occurrence nets-we have:

(i) $g\left(a^{F}\right)=\bigoplus_{i \in I_{a}} b_{i}^{F}$, for some index set $I_{a}$;

(ii) $\pi_{a} \circ g_{i}^{o p} \circ i n_{b_{i}}$ is a block function, where

$\pi_{a}$ is the projection of $\{a\} \times\left[n_{a}\right]$ to $\left[n_{a}\right]$,

$\pi_{a}^{-1}$ is the inverse bijection from $\left[n_{a}\right]$ to $\{a\} \times\left[n_{a}\right]$, and 


$$
g_{i}^{o p}:\left\{b_{i}\right\} \times\left[n_{b_{i}}\right] \rightarrow\{a\} \times\left[n_{a}\right] \text { is } g_{x}^{o p} \text { restricted to }\left\{b_{i}\right\} \times\left[n_{b_{i}}\right] .
$$

The composition in (ii) can be summarized by means of the diagram

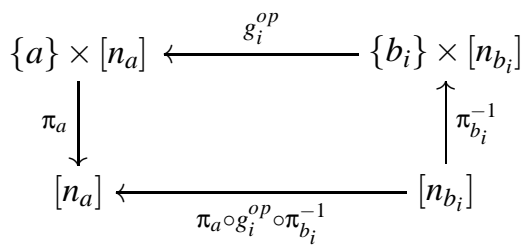

This, with the obvious componentwise composition of morphisms, defines the category DecOcc.

A family is thus a collection of finitely many places with the same pre-set, and a decorated occurrence net is an occurrence net where each place belongs to exactly one family. Families, and therefore decorated occurrence nets, are capable of describing relationships between places by grouping them together. We shall use families to relate places which are instances of the same place obtained in a process of unfolding. Morphisms treat families in a special way: they map families to families (condition $i$ ) and they do that in a unique predetermined way (condition $i i$ ). This allows us to focus exactly on the fact that, say, $a^{F}$ is mapped to $b^{F}$. Since the way to map a family to another family is fixed by definition, in the following we will often define morphisms just by saying which families are sent to which families.

Observe that the full subcategory of DecOcc consisting of the nets $\Theta$ with $S_{\Theta}=\bigcup_{a \in A_{\Theta}}\{a\} \times$ [1] is (isomorphic to) Occ. Observe also that, since the initial marking consists exactly of the elements with empty pre-set and, by point (ii) in Definition 3.2, elements of a family have the same pre-set, for a decorated occurrence net $u_{\Theta}$ is of the form $\bigoplus_{i \in I} a_{i}^{F}$.

The following is a useful property of decorated occurrence net morphisms which directly follows from their definition.

Proposition 3.3. (Decorated Occurrence Net Morphisms) Let $\Theta_{0}$ and $\Theta_{1}$ be decorated occurrence nets and $\langle f, g\rangle: \Theta_{0} \rightarrow \Theta_{1}$ a morphism in DecOcc. Then

$$
\begin{aligned}
& \forall\left[\left[b^{F}\right]\right] \subseteq\left[\left[u_{N_{1}}\right]\right], \exists !\left[[ a ^ { F } ] \subseteq \left[[ u _ { N _ { 0 } } ] \text { such that } \left[[ b ^ { F } ] \subseteq \left[\left[g\left(a^{F}\right)\right]\right.\right.\right.\right. \text { and } \\
& \forall t \in T_{\Theta_{0}}, \forall\left[\left[b^{F}\right]\right] \subseteq\left[\partial_{N_{1}}^{1}(f(t))\right], \exists !\left[[ a ^ { F } ] \subseteq \left[[ \partial _ { N _ { 0 } } ^ { 1 } ( t ) ] \text { such that } \left[\left[b^{F}\right] \subseteq\left[g\left(a^{F}\right)\right] .\right.\right.\right.
\end{aligned}
$$

We have seen that for occurrence nets and decorated occurrence nets simple concepts of causal dependence $(\prec)$ and conflict (\#) can be defined. The orthogonal concept is that of concurrency.

Definition 3.4. (Concurrent Elements) Given a (decorated) occurrence net $\Theta$ (which defines $\prec$, $\preceq$ and \#), we can define

- for $x, y \in T_{\Theta} \cup S_{\Theta}, x$ co $y$ if it is not the case that $(x \prec y$ or $y \prec x$ or $x \# y)$;

- for $X \subseteq T_{\Theta} \cup S_{\Theta}, \operatorname{Co}(X)$ if $\forall x, y \in X, x$ co $y$, and $\left|\left\{t \in T_{\Theta} \mid \exists x \in X, t \preceq x\right\}\right| \in \omega$.

As a first step in relating the categories DecOcc and PTNets, we define a functor from decorated occurrence nets to PT nets.

Definition 3.5. $\left((-)^{+}\right.$: from DecOcc to PTNets) For $\Theta$ a decorated occurrence net, let the map $\left({ }_{-}\right)^{+}: S_{\Theta}^{\mathcal{M}_{\infty}} \rightarrow A_{\Theta}^{\mathcal{M}_{\infty}}$ be the ()$^{\mathcal{M}_{\infty}}$-homomorphism such that $(a, j)^{+}=a$. Then, define $\Theta^{+}$to be the net $\left((-)^{+} \circ \partial_{\Theta}^{0},(-)^{+} \circ \partial_{\Theta}^{1}:\left(T_{\Theta}, 0\right) \rightarrow A_{\Theta}^{\mathcal{M}}, u_{\Theta}^{+}\right)$.

For a morphism $\langle f, g\rangle: \Theta_{0} \rightarrow \Theta_{1}$, let $\langle f, g\rangle^{+}: \Theta_{0}^{+} \rightarrow \Theta_{1}^{+}$be $\left\langle f,\left({ }_{-}\right)^{+} \circ g \circ \rho\right\rangle$ where $\rho: A_{\Theta_{0}}^{\mathcal{M}_{\infty}} \rightarrow S_{\Theta_{0}}^{\mathcal{M}_{\infty}}$ is the $\left({ }_{-}\right)^{\mathcal{M}}$-homomorphism such that $\rho(a)=(a, 1)$. 
The following example shows the result of applying $\left({ }_{-}\right)^{+}$to a decorated occurrence net. In all the pictures to follow, a family is represented by drawing its elements from left to right in accordance with its ordering, and enclosing them into an oval. Families of cardinality one are not explicitly indicated.

Example 3.6.
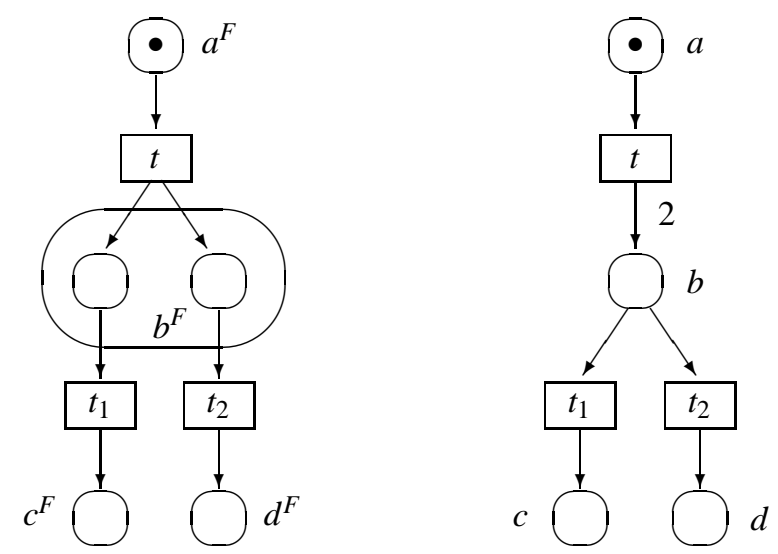

A decorated occurrence net $\Theta$ and the net $\Theta^{+}$

Proposition 3.7. $\left((-)^{+}\right.$is well-defined $) \Theta^{+}$is a PT net and $\langle f, g\rangle^{+}$is a PT net morphism.

Proof. The first statement is completely clear. Let us show the second. Conditions $(i)$ and (ii) are trivial while condition $(v)$ derives directly from Proposition 3.3. Observe that, if $g(a, i)=$ $\bigoplus_{l}\left(\bigoplus\left\{b_{l}\right\} \times\left[k_{l}\right]_{i}\right)$, then, by definition of $g$, we have $g(a, j)=\bigoplus_{l}\left(\bigoplus\left\{b_{l}\right\} \times\left[k_{l}\right]_{j}\right)$ and thus $g(a, i)^{+}=\bigoplus_{l} k_{l} b^{l}=g(a, j)^{+}$. Therefore, $g(u)^{+}=\left(\left({ }_{-}\right)^{+} \circ g \circ \rho\right)\left(u^{+}\right)$.

(iii). $\left(()^{+} \circ g \circ \rho\right)\left(u_{\Theta_{0}^{+}}\right)=\left(()^{+} \circ g \circ \rho\right)\left(u_{\Theta_{0}}^{+}\right)=g\left(u_{\Theta_{0}}\right)^{+}=u_{\Theta_{1}}^{+}=u_{\Theta_{1}^{+}}$.

(iv). $\quad\left((-)^{+} \circ g \circ \rho\right)\left(\partial_{\Theta_{0}^{+}}^{i}(t)\right)=\left((-)^{+} \circ g \circ \rho\right)\left(\partial_{\Theta_{0}}^{i}(t)^{+}\right)$

$$
=g\left(\partial_{\Theta_{0}}^{i}(t)\right)^{+}=\partial_{\Theta_{1}}^{i}(f(t))^{+}=\partial_{\Theta_{1}^{+}}^{i}(f(t)) .
$$

Proposition 3.8. $\left(()^{+}\right.$: DecOcc $\rightarrow$ PTNets $)()^{+}$: DecOcc $\rightarrow$ PTNets is a functor.

Proof. Clearly, $\left\langle i d_{T_{\Theta}}, i d_{S_{\Theta}}\right\rangle^{+}=\left\langle i d_{T_{\Theta}}, i d_{A_{\Theta}}\right\rangle$. Moreover, given $\langle h, k\rangle \circ\langle f, g\rangle: \Theta_{0} \rightarrow \Theta_{1}$, we have that for each $u \in A_{\Theta_{0}}^{\mathcal{M}_{\infty}}$

$$
\begin{aligned}
\left((-)^{+} \circ k \circ \rho\right) \circ\left((-)^{+} \circ g \circ \rho\right)(u) & =\left((-)^{+} \circ k \circ \rho\right)\left((g \circ \rho)(u)^{+}\right) \\
& =k((g \circ \rho)(u))^{+}=\left((-)^{+} \circ(k \circ g) \circ \rho\right)(u) .
\end{aligned}
$$

So, $(\langle h, k\rangle \circ\langle f, g\rangle)^{+}=\langle h, k\rangle^{+} \circ\langle f, g\rangle^{+}$.

Nets obtained via $\left({ }^{-}\right)^{+}$from decorated occurrence nets have a structure very similar to that of occurrence nets. We will denote by $\mathrm{DecOcc}^{+}$the full subcategory of PTNets consisting of (nets isomorphic to) nets of the form $\Theta^{+}$.

Proposition 3.9. (Structure of Decorated Occurrence Nets) If $\Theta$ is a decorated occurrence net, then $\Theta^{+}$is a PT net such that:

(i) $a \in\left[\left[u_{\Theta^{+}}\right]\right]$if and only if $\bullet a=\varnothing$; 
(ii) $\forall a \in S_{\Theta^{+}},|\bullet a| \leq 1$;

(iii) the relation $\prec$ is irreflexive and $\forall t \in T_{\Theta^{+}},\left\{t^{\prime} \in T_{\Theta^{+}} \mid t^{\prime} \prec t\right\}$ is finite.

Moreover, if $\Theta$ is (isomorphic to) an occurrence net, $\Theta^{+}$is an occurrence net isomorphic to $\Theta$.

Let $\mathcal{B}$ range over Occ, DecOcc and DecOcc ${ }^{+}$. Thanks to the stratified structure of the nets in $\mathcal{B}$, for them we can define the concepts of depth of elements and, consequently, of subnet of depth $n$. Essentially, this will allow us to work on such nets by induction.

Definition 3.10. (Depth) Let $\Theta$ be a net in $\mathcal{B}$. The depth of elements in $T_{\Theta} \cup S_{\Theta}$ is defined inductively by:

$-\operatorname{depth}(b)=0 \quad$ if $b \in\left[\left[u_{\Theta}\right]\right]$;

- $\operatorname{depth}(b)=\operatorname{depth}(t) \quad$ if $\{t\}=\bullet b$;

- $\operatorname{depth}(t)=\max \{\operatorname{depth}(b) \mid b \prec t\}+1$.

Definition 3.11. (Subnets of a Net) Given a net $\Theta$ in $\mathcal{B}$ define its subnet of depth $n, \Theta^{(n)}$, as

$-T_{\Theta(n)}=\left\{t \in T_{\Theta} \mid \operatorname{depth}(t) \leq n\right\} ;$

$-S_{\Theta^{(n)}}=\left\{b \in S_{\Theta} \mid \operatorname{depth}(b) \leq n\right\}$;

- $\partial_{\Theta^{(n)}}^{0}$ and $\partial_{\Theta}^{1}{ }_{\Theta}^{(n)}$ are the restrictions of $\partial_{\Theta}^{0}$ and $\partial_{\Theta}^{1}$ to $T_{\Theta^{(n)}}$;

$-u_{\Theta(n)}=u_{\Theta}$.

Clearly, $\Theta^{(n)}$ is a net in $\mathcal{B}$, whenever $\Theta$ is such. For each $n \leq m$ there exists a morphism $i n_{n, m}: \Theta^{(n)} \rightarrow \Theta^{(m)}$ whose components are both set inclusions. In the following we shall call such net morphisms simply inclusions. Observe that, if $\langle f, g\rangle: \Theta_{0} \rightarrow \Theta_{1}$ is an inclusion, we obviously have $u_{\Theta_{0}}=u_{\Theta_{1}}$ and, for each $t \in T_{\Theta_{0}}, \partial_{\Theta_{0}}^{i}(t)=\partial_{\Theta_{1}}^{i}(t), i=0,1$.

The sequence of nets $\Theta^{(n)}, n \in \omega$, can be seen as a sequence of finite approximations which, together with the corresponding inclusions, determines $\Theta$ uniquely (up to isomorphisms). We shall formalize this intuition by means of the categorical notion of colimit. The following results will allow us in Section 4 to define the unfolding of a PT net $N$ in terms of finite unfoldings, viz. its subnets of depth $n$. We first need to show that $\mathcal{B}$ possesses the required colimits. Consider the category $\omega=\{0 \rightarrow 1 \rightarrow 2 \rightarrow 3 \cdots\}$ and the class $\mathcal{D}$ of diagrams $D: \omega \rightarrow \mathcal{B}$ such that $D(n \rightarrow$ $n+1)=i n_{n}: D(n) \rightarrow D(n+1)$ is an inclusion. For such a class we have the following results. The reader is referred to (MacLane 1971, III.3) for the definition of the categorical concepts involved.

Proposition 3.12. (Colim $(D)$ exists) For any $D \in \mathcal{D}$, the colimit of $D$ in $\mathcal{B}$ exists.

Proof. Consider the net $\Theta=\left(\partial_{\Theta}^{0}, \partial_{\Theta}^{1}:\left(T_{\Theta}, 0\right) \rightarrow S_{\Theta}^{\mathcal{M}}, u_{\Theta}\right)$ where

$$
\begin{array}{r}
T_{\Theta}=\bigcup_{n} T_{D(n)} \quad S_{\Theta}=\bigcup_{n} S_{D(n)} \quad u_{\Theta}=u_{D(0)} \\
\partial_{\Theta}^{i}(t)=\partial_{D(n)}^{i}(t) \text { for any } n \text { such that } t \in T_{D(n)} .
\end{array}
$$

Clearly, $\Theta$ is well-defined, is a net, and belongs to $\mathcal{B}$.

Now, for any $n$, let $\mu_{n}: D(n) \rightarrow \Theta$ be the obvious inclusion. By definition we have $\mu_{n}=\mu_{n+1} \circ i n_{n}$. Now consider a family of morphisms $\tau_{n}: D(n) \rightarrow \Theta^{\prime}, n \in \omega$, such that $\tau_{n}=\tau_{n+1} \circ i n_{n}$. Define $\sigma: \Theta \rightarrow \Theta^{\prime}$ as follows.

$$
\begin{aligned}
& \sigma(t)=\tau_{n}(t) \text { for } n \text { such that } t \in T_{D(n)} \\
& \sigma(a)=\tau_{n}(a) \text { for } n \text { such that } a \in S_{D(n)} .
\end{aligned}
$$


Clearly, $\sigma$ is a morphism in $\mathcal{B}$. Now consider $\sigma \circ \mu_{n}: D(n) \rightarrow \Theta^{\prime}$. We have that, for any $t \in T_{D(n)}$, $\sigma(t)=\tau_{n}(t)$ and for any $a \in S_{D(n)}, \sigma(a)=\tau_{n}(a)$. Therefore, since $\mu_{n}$ is an inclusion, we have $\sigma \circ \mu_{n}=\tau_{n}$ for each $n \in \omega$.

Given any $\sigma^{\prime}: \Theta \rightarrow \Theta^{\prime}$, suppose that $\sigma^{\prime} \circ \mu_{n}=\tau_{n}$ for each $n \in \omega$. Since $t \in T_{\Theta}\left(a \in S_{\Theta}\right)$ belongs to $T_{D(n)}\left(\right.$ to $\left.S_{D(n)}\right)$ for some $n$, we have that $\sigma^{\prime}(t)=\tau_{n}(t)=\sigma(t)\left(\sigma^{\prime}(a)=\tau_{n}(a)=\sigma(a)\right)$. Therefore, $\sigma^{\prime}=\sigma$.

Proposition 3.13. ( $\Theta$ is the colimit of its subnets) Given a net $\Theta$ in $\mathcal{B}$, let $D_{\Theta}: \omega \rightarrow \mathcal{B}$ be the functor such that $D_{\Theta}(n)=\Theta^{(n)}$ and $D_{\Theta}(n \rightarrow n+1)=i n_{n, n+1}: \Theta^{(n)} \rightarrow \Theta^{(n+1)}$. Then $\Theta=\operatorname{Colim}\left(D_{\Theta}\right)$.

Proof. Since $D_{\Theta} \in \mathcal{D}$, we are in the conditions of the previous proposition. So, it is enough to observe that the colimit construction for diagrams in $\mathcal{D}$ in the proof of that proposition gives a family $\mu_{n}: D(n) \rightarrow \Theta, n \in \omega$, where $\mu_{n}: \Theta^{(n)} \rightarrow \Theta$ is the inclusion of $\Theta^{(n)}$ in $\Theta$.

The next proposition shows that ()$\left.^{+}\right)^{+}$behaves well with respect to $\mathcal{D}$, namely to apply (_) ${ }^{+}$to $\Theta$ in DecOcc is to apply it pointwise to $D_{\Theta}$ and then take the colimit in $\mathrm{DecOcc}^{+}$of the resulting diagram.

Proposition 3.14. $\left(()^{+}\right.$preserves the colimit of $\left.D_{\Theta}\right)$ If $\mathcal{B}=\operatorname{DecOcc}$, then $\operatorname{Colim}\left(D_{\Theta}\right)^{+}=$ $\operatorname{Colim}\left(D_{\Theta}^{+}\right)$.

Proof. Since the previous proposition states that each $\Theta$ is completely identified by the diagram $D_{\Theta}$, it is enough to observe that $D_{\Theta}^{+}$is exactly $D_{\Theta^{+}}$. So, $\operatorname{Colim}\left(D_{\Theta}^{+}\right)=\operatorname{Colim}\left(D_{\Theta^{+}}\right)=$ $\Theta^{+}=\operatorname{Colim}\left(D_{\Theta}\right)^{+}$.

\section{PT Net Unfoldings}

In this section, we define the unfolding of PT nets in terms of decorated occurrence nets and show that it is a functor from PTNets to DecOcc which is right adjoint to $(-)^{+}$.

We start by giving the object component of such a functor. To this end, given a net $N$, we define a sequence of decorated occurrence nets, whose $n$th element approximates the unfolding of $N$ up to depth $n$, i.e., it reflects the behaviour of the original net up to step sequences of length at most $n$. Clearly, the unfolding of $N$ will be defined as the colimit of an appropriate $\omega$-diagram built on the sequence of approximating nets.

The purpose of the following inductive definition is to generate all the possible instances of places and transitions of $N$ by decorating them with their 'history'. The families of places in the approximating nets represent instances of places of $N$ with the same history. Precisely, they are pairs $(x, b)$, where $b \in S_{N}$ and $x$ is a set encoding the history of these instances of $b$. Therefore, the places are triples $((x, b), i), i$ determining the position of the place in its family. Analogously, the transitions are pairs $(B, t)$ where $t \in T_{N}$ and the set $B$ represents the history of the instance of $t$.

Definition 4.1. (PT Nets Unfoldings: $\left.\mathcal{U}[]^{(k)}\right)$ Let $N=\left(\partial_{N}^{0}, \partial_{N}^{1}:\left(T_{N}, 0\right) \rightarrow S_{N}^{\mathscr{M}}, u_{N}\right)$ be a net in PTNets. We define the nets $\mathcal{u}[N]^{(k)}=\left(\partial_{k}^{0}, \partial_{k}^{1}:\left(T_{k}, 0\right) \rightarrow S_{k}^{\mathscr{M}}, u_{k}\right)$, for $k \in \omega$, where:

$-S_{0}=\bigcup\left\{\{(\varnothing, b)\} \times[n] \mid u_{N}(b)=n\right\} ;$

- $T_{0}=\{0\}$, and the $\partial_{0}^{i}$ with the obvious definitions;

$-u_{0}=\bigoplus S_{0}$;

for $k>0$, 


$$
\begin{aligned}
& -\frac{B=\left\{\left(\left(x_{j}, b_{j}\right), i_{j}\right) \mid j \in J\right\} \subseteq S_{k-1}, \operatorname{Co}(B), t \in T_{N}, \bigoplus_{j \in J} b_{j}=\partial_{N}^{0}(t)}{(B, t) \in T_{k} \text { and } \partial_{k}^{0}(B, t)=\bigoplus B} \\
& -\frac{t_{0}=(B, t) \in T_{k}, \partial_{N}^{1}(t)=\bigoplus_{j \in J} n_{j} b_{j}}{\left\{\left(\left\{t_{0}\right\}, b_{j}\right)\right\} \times\left[n_{j}\right] \subseteq S_{k}, \forall j \in J, \quad \text { and } \partial_{k}^{1}\left(t_{0}\right)=\bigoplus_{j, 1 \leq i \leq n_{j}}\left(\left(\left\{t_{0}\right\}, b_{j}\right), i\right)} \\
& -u_{k}=\bigoplus_{j}\left(\left(\varnothing, b_{j}\right), i_{j}\right)=\bigoplus S_{0}=u_{0} .
\end{aligned}
$$

Therefore, informally speaking, the net $\mathcal{U}[N]^{(0)}$ is obtained by exploding in families the initial marking of $N$, and $\mathcal{U}[N]^{(n+1)}$ is obtained, inductively, by generating a new transition for each possible subset of concurrent places of $\mathcal{U}[N]^{(n)}$ whose corresponding multiset of places of $N$ constitutes the source of some transition $t$ of $N$; the target of $t$ is also exploded in families which are added to $\mathcal{U}[N]^{(n+1)}$. Clearly, we shall define $\mathcal{U}[N]$ as the colimit of the sequence of the $\mathcal{u}[N]^{(n)}, n \in \omega$. To do that, we first need to prove the following lemma.

Lemma 4.2. $\left(\mathcal{U}[N]^{(n)}\right.$ is a decorated occurrence net) For all $n \in \omega, \mathcal{U}[N]^{(n)}$ is a decorated occurrence net of depth $n$. Moreover, for each $n \in \omega$ there is an inclusion $i n_{n}: \mathcal{U}[N]^{(n)} \rightarrow \mathcal{U}[N]^{(n+1)}$.

Proof. That $\mathcal{U}[N]^{(n)}$ has depth $n$ and that there exists an inclusion from $\mathcal{U}[N]^{(n)}$ to $\mathcal{U}[N]^{(n+1)}$ is obvious from the definition. We have to show that $\mathcal{u}[N]^{(n)}$ is a decorated occurrence net. For each $t \in T_{n}, \partial_{n}^{i}(t)$ is a multiset where all the elements have multiplicity one, i.e., a set. The same happens for $u_{n}$.

(i) Observe that for each $((x, b), i) \in S_{n}, \bullet((x, b), i)=x$ which is the empty set or a singleton. So $\left.\right|^{\bullet}((x, b), i) \mid \leq 1$.

(ii) Moreover, $((x, b), i) \in\left[\left[u_{n}\right]\right]$ iff $x=\varnothing$ iff $\bullet((x, b), i)=\varnothing$.

(iii) By definition of $\mathcal{u}[N]^{(n)}$, whenever $x \prec^{1} y \prec^{1} z$, depth $(z)=\operatorname{depth}(x)+1$. Since $x, z \in T_{n}$ or $x, z \in S_{n}$ implies that there exists at least one $y$ such that $x \prec y \prec^{1} z$ we have $\operatorname{depth}(x)<$ $\operatorname{depth}(z)$. So $x \neq z$ and $\prec$ is irreflexive. This, together with $(i)$ and (ii), implies that, in each reachable marking, every place has multiplicity at most one. In fact, since that happens in $u_{n}$, since each place has only one pre-event and each transition occurs at most once in any computation, there is no way to generate multiple tokens in a place. Moreover, $\forall t \in T_{n},\left\{t^{\prime} \in T_{n} \mid t^{\prime} \prec t\right\}$ is finite, because of the definition of Co.

(iv) \# is irreflexive. Recall that $x \# x$ iff $\exists t, t^{\prime} \in T_{n}, t \neq t^{\prime}$ and $t \#_{m} t^{\prime}$ such that $t \preceq_{n} x$ and $t^{\prime} \preceq_{n} x$. So, by $(i), x$ cannot be a place, otherwise we would have backward branching. This means that there exist $b, b^{\prime} \in\left[\left[\partial_{n}^{0}(x)\right]\right], b \neq b^{\prime}$ such that $b$ co $b^{\prime}$, i.e., $x=(B, t)$ and not $\operatorname{Co}(B)$. This is impossible.

The other conditions of decorated occurrence nets obviously hold.

Definition 4.3. (PT Net Unfoldings: $\mathcal{u}[-]$ ) We define $\mathcal{u}[N]$ to be the colimit of the diagram $D: \omega \rightarrow$ DecOcc such that $D(n)=\mathcal{U}[N]^{(n)}$ and $D(n \rightarrow n+1)=i n_{n}$. By Lemma $4.2 D$ belongs to $\mathcal{D}$ and so, by Proposition 3.12, the colimit exists and is a decorated occurrence net. 


\section{Example 4.4.}
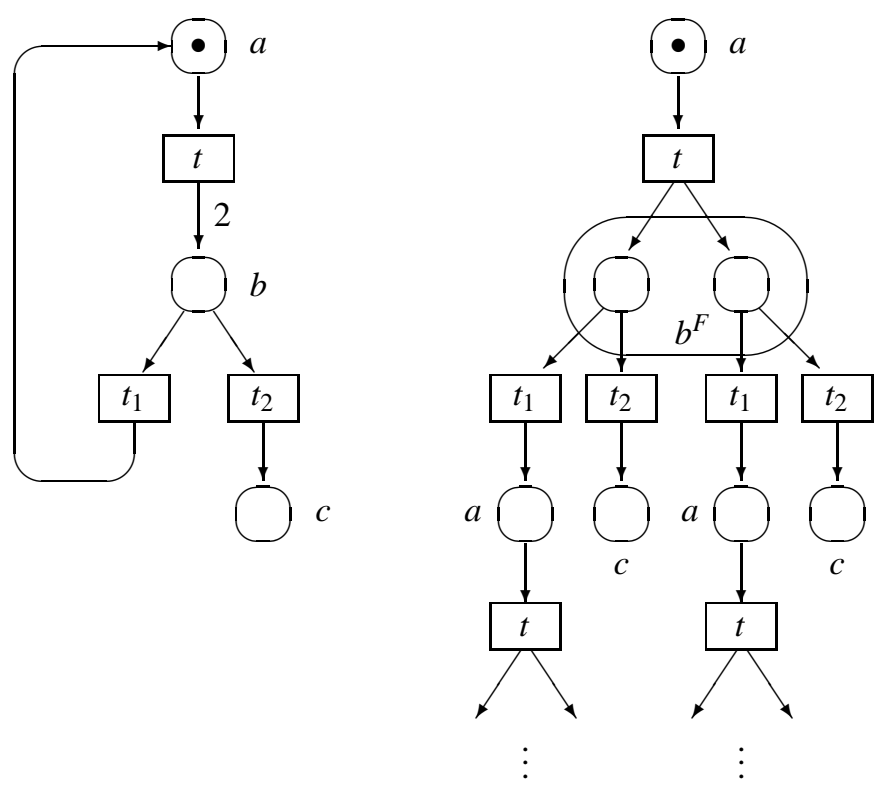

A PT Net $N$ and (part of) its unfolding $\mathcal{u}[N]$

The correspondence between elements of the unfolding and elements of the original net is formalized by the folding morphism, which will also be the counit of the adjunction.

Proposition 4.5. (Folding Morphism) Consider the map $\varepsilon_{N}=\left\langle f_{\varepsilon}, g_{\varepsilon}\right\rangle: \mathcal{U}[N]^{+} \rightarrow N$ defined by

$-f_{\varepsilon}(B, t)=t$ and $f_{\varepsilon}(0)=0$;

$-g_{\varepsilon}\left(\bigoplus_{i}\left(x_{i}, y_{i}\right)\right)=\bigoplus_{i} y_{i}$.

Then, $\varepsilon_{N}$ is a morphism in PTNets, called the folding of $\mathcal{U}[N]$ into $N$.

Proof. Since the transitions of $\mathcal{u}[N]^{+}$are of the form $t_{0}=(B, t):(\bigoplus B)^{+} \rightarrow(\bigoplus C)^{+}$, where $\left.B=\left\{\left(\left(x_{j}, b_{j}\right), i_{j}\right) \mid j \in J\right\} \subseteq S_{\mathcal{u}[N]}, C=\left\{\left(\left\{t_{0}\right\}, c_{k}\right), i_{k}\right) \mid k \in K\right\}, t \in T_{N}, \bigoplus_{j \in J} b_{j}=\partial_{N}^{0}(t)$, and $\bigoplus_{k \in K} c_{k}=\partial_{N}^{1}(t)$, we immediately obtain

$$
g_{\varepsilon}\left(\partial_{\mathcal{u}[N]^{+}}^{i}(B, t)\right)=\partial_{N}^{i}\left(f_{\varepsilon}(B, t)\right) .
$$

Since $u_{\mathcal{U}[N]^{+}}=\bigoplus_{b \in S_{N}} u_{N}(b) \cdot(\varnothing, b)$, we have $g_{\varepsilon}\left(u_{\mathcal{U}[N]^{+}}\right)=\bigoplus_{b \in S_{N}} u_{N}(b) \cdot b=u_{N}$. Concerning condition $(v)$ in Definition 1.1, observe that $\left[\left[g_{\varepsilon}(x, a)\right] \cap\left[\left[g_{\varepsilon}(y, b)\right] \neq \varnothing\right.\right.$ implies $a=b$. So, if $(x, a) \neq(y, b)$, then $(x, a) \notin\left[\left[u_{\mathcal{u}[N]^{+}}\right]\right]$or $(y, b) \notin\left[\left[u_{\mathcal{u}[N]^{+}}\right]\right]$, because either $x$ or $y$ must be nonempty, and $\bullet(x, a) \cap \bullet(y, b)=x \cap y=\varnothing$.

The next lemma is the final ingredient we need to prove that $\mathcal{u}[-]$ is right adjoint to $(-)^{+}$.

Lemma 4.6. (Occurrence Net Morphisms preserve Concurrency) Let $\Theta_{0}$ and $\Theta_{1}$ be (decorated) occurrence nets and let $\langle f, g\rangle: \Theta_{0} \rightarrow \Theta_{1}$ be a morphism. Then, for each $t_{0} \in T_{\Theta_{0}}$, we have $\operatorname{Co}\left(\left[\left[\partial_{\Theta_{0}}^{0}\left(t_{0}\right)\right]\right]\right)$ and $\operatorname{Co}\left(\left[\left[g\left(\partial_{\Theta_{0}}^{0}\left(t_{0}\right)\right)\right]\right]\right)$.

Proof. Since, by definition of (decorated) occurrence nets, $\left\{t^{\prime} \preceq t\right\}$ is finite, we have not $\operatorname{Co}\left(\left[\left[\partial_{\Theta_{0}}^{0}\left(t_{0}\right)\right]\right]\right)$ iff $\exists b, b^{\prime} \in\left[\left[\partial_{\Theta_{0}}^{0}\left(t_{0}\right)\right]\right]$ such that $b \# b^{\prime}$. This would mean that $\exists t, t^{\prime} \in T_{\Theta_{0}}, t \neq t^{\prime}$ and 
$t \#_{m} t^{\prime}$ such that $t \preceq b$ and $t^{\prime} \preceq b^{\prime}$. Thus, since $t \preceq t_{0}$ and $t^{\prime} \preceq t_{0}$, we would have $t_{0} \# t_{0}$ which is impossible since $\Theta_{0}$ is a (decorated) occurrence net. Furthermore, $g\left(\partial_{\Theta_{0}}^{0}\left(t_{0}\right)\right)=\partial_{\Theta_{1}}^{0}\left(f\left(t_{0}\right)\right)$, which is the pre-set of a transition of a (decorated) occurrence net and so, by the first part of this proposition, $\operatorname{Co}\left(\left[\left[g\left(\partial_{\Theta_{0}}^{0}\left(t_{0}\right)\right)\right]\right]\right)$.

Theorem 4.7. $\left(\left({ }_{-}\right)^{+} \dashv \mathfrak{u}\left[\left[_{-}\right]\right)\right.$The pair $\left\langle\left({ }_{-}\right)^{+}, \mathfrak{u}\left[\left[_{-}\right]\right\rangle:\right.$DecOcc $\rightarrow$ PTNets constitutes an adjunction.

Proof. Let $N$ be a PT Net and $\mathcal{u}[N]$ its unfolding. By (MacLane 1971, Theorem 2, pg. 81), it is enough to show that the folding $\varepsilon_{N}: \mathfrak{U}[N]^{+} \rightarrow N$ is universal from (_) ${ }^{+}$to $N$, i.e., for any decorated occurrence net $\Theta$ and any morphism $k: \Theta^{+} \rightarrow N$ in PTNets, there exists a unique $h: \Theta \rightarrow \mathcal{U}[N]$ in DecOcc such that $k=\varepsilon_{N} \circ h^{+}$.

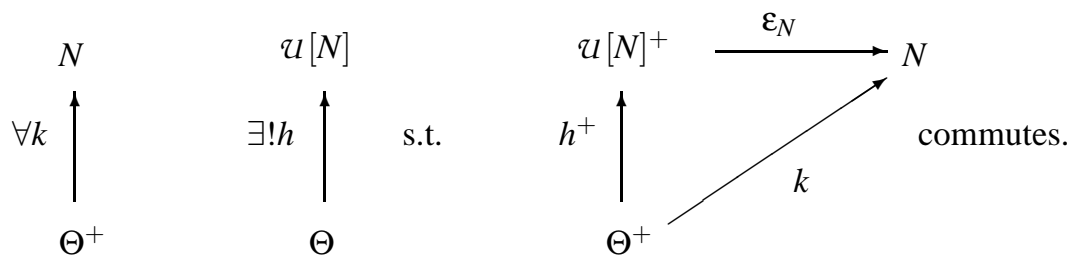

Consider the diagram in DecOcc given by $D_{\Theta}(n)=\Theta^{(n)}$, the subnet of $\Theta$ of depth $n$ and $D_{\Theta}(n \rightarrow$ $n+1)=i n_{n}: \Theta^{(n)} \rightarrow \Theta^{(n+1)}$. We define a sequence of morphisms of nets $h_{n}: \Theta^{(n)} \rightarrow \mathcal{U}[N]$, such that for each $n, h_{n}=h_{n+1} \circ i n_{n}$. Since $\Theta=\operatorname{Colim}\left(D_{\Theta}\right)$, there is a unique $h: \Theta \rightarrow \mathcal{U}[N]$ such that $h \circ \mu_{n}=h_{n}$ for each $n$. At the same time, we show that

$$
\forall n \in \omega, k \circ \mu_{n}^{+}=\varepsilon_{N} \circ h_{n}^{+}
$$

and that the $h_{n}$ form the unique sequence of morphisms $h_{n}: \Theta^{(n)} \rightarrow \mathfrak{U}[N]$ such that (1) holds. Now, by functoriality of $(-)^{+}$, we have that

$$
\forall n \in \omega, k \circ \mu_{n}^{+}=\varepsilon_{N} \circ h^{+} \circ \mu_{n}^{+} .
$$

Then, since by Proposition $3.14\left({ }_{-}\right)^{+} \circ D_{\Theta}=D_{\Theta^{+}}$, and, by Proposition 3.13, $\Theta^{+}=\operatorname{Colim}\left(D_{\Theta}^{+}\right)=$ $\operatorname{Colim}\left(()^{+} \circ D_{\Theta}\right)$, by the universal property of the colimit we must have $k=\varepsilon_{N} \circ h^{+}$. To show the uniqueness of $h$, let $h^{\prime}$ be such that $k=\varepsilon_{N} \circ h^{\prime}$. Then we have $k \circ \mu_{n}^{+}=\varepsilon_{N} \circ h^{\prime} \circ \mu_{n}^{+}$. But $h_{n}$ is the unique morphism for which this happens. Therefore, for each $n, h_{n}=h^{\prime} \circ \mu_{n}$ and so, by the universal property of the colimit, $h=h^{\prime}$.

Let us now define $h_{n}$ and therefore $h: \Theta \rightarrow \mathcal{U}[N]$, and show that the $h_{n}, n \in \omega$, form the unique sequence of morphisms for which (1) above holds.

depth 0. Suppose that $u_{\Theta^{+}}=\bigoplus_{i} n_{i} a_{i}$. So $u_{\Theta}=\bigoplus_{i}\left(\bigoplus\left\{a_{i}\right\} \times\left[n_{i}\right]\right)$. Assume further that $k\left(a_{j}\right)=$ $\bigoplus_{l} m_{l}^{j} b_{l}^{j}$. By definition of $k$, since $k$ does not merge different places in the initial marking and $k\left(u_{\Theta^{+}}\right)=u_{N}$, we have $u_{N}=v \oplus \bigoplus_{l} n_{j} m_{l}^{j} b_{l}^{j}$, with $b_{l}^{j} \notin[[v]]$. Thus, in $\mathcal{U}[N]$ we have the places $\bigcup_{l}\left\{\left(\varnothing, b_{l}^{j}\right)\right\} \times\left[n_{j} m_{l}^{j}\right]$. So, we define

$$
h_{0}\left(a_{j}, i\right)=\bigoplus_{l}\left(\bigoplus\left\{\left(\varnothing, b_{l}^{j}\right)\right\} \times\left[m_{l}^{j}\right]_{i}\right) .
$$

We have

$$
\begin{aligned}
h_{0}^{+}\left(a_{j}\right) & =\left(h_{0}\left(a_{j}, i\right)\right)^{+}=\bigoplus_{j} m_{l}^{j}\left(\varnothing, b_{l}^{j}\right) \text { and } \\
\varepsilon_{N} \circ h_{0}^{+}\left(a_{j}\right) & =\bigoplus_{l} m_{l}^{j} b_{l}^{j}=k\left(a_{j}\right)=k \circ \mu_{0}^{+}\left(a_{j}\right) .
\end{aligned}
$$


Observe that $h_{0}$ so defined, lifting its place component to a $\left({ }_{-}\right)^{\mathcal{M}_{\infty}}$-homomorphism, is a morphism $\Theta^{(0)} \rightarrow \mathcal{U}[N]$ and that it is completely determined by $k$ and the conditions of decorated occurrence net morphisms.

depth $\mathrm{n}+1$. Let us suppose that we have defined $h_{n}: \Theta^{(n)} \rightarrow \mathcal{U}[N]$ and that it is a morphism. Suppose that for each $m \leq n, h_{m}$ is the unique morphism such that $\varepsilon_{N} \circ h_{m}^{+}=k \circ \mu_{m}$. Let $h_{n+1}$ be $h_{n}$ on the elements of depth less or equal to $n$. Now, we define $h_{n+1}$ on the elements of depth $n+1$. Let $t_{1} \in T_{\Theta}$ such that $\operatorname{depth}\left(t_{1}\right)=n+1$ and $k\left(t_{1}\right)=t$. Since $\left[\left[\partial_{\Theta}^{0}\left(t_{1}\right)\right]\right]$ is a set of elements of depth less or equal to $n, h_{n}\left(\partial_{\Theta}^{0}\left(t_{1}\right)\right)$ is defined. Since $h_{n}$ is a morphism, by Lemma 4.6, we have $\operatorname{Co}\left(\left[\left[h_{n}\left(\partial_{\Theta}^{0}\left(t_{1}\right)\right)\right]\right]\right)$. Moreover, since $\varepsilon_{N} \circ h_{n}^{+}=k \circ \mu_{n}^{+}$, we have that

$$
\begin{aligned}
\partial_{N}^{0}(t)=k\left(\partial_{\Theta}^{0}(t)\right)= & \varepsilon_{N} \circ h_{n}^{+}\left(\partial_{\Theta^{+}}^{0}\left(t_{1}\right)\right)=\varepsilon_{N} \circ h_{n}^{+}\left(\left(\partial_{\Theta}^{0}\left(t_{1}\right)\right)^{+}\right) \\
= & \varepsilon_{N} \circ h_{n}\left(\partial_{\Theta}^{0}\left(t_{1}\right)\right)^{+}=\bigoplus_{j \in J} b_{j}, \\
& \quad \text { for } J \text { such that }\left\{\left(\left(x_{j}, b_{j}\right), i_{j}\right) \mid j \in J\right\}=\left[\left[h_{n}\left(\partial_{\Theta}^{0}\left(t_{1}\right)\right)\right] . .\right.
\end{aligned}
$$

Therefore $t_{0}=\left(\left[\left[h_{n}\left(\partial_{\Theta}^{0}\left(t_{1}\right)\right)\right]\right], t\right)=\left(\left[\left[h_{n+1}\left(\partial_{\Theta}^{0}\left(t_{1}\right)\right)\right]\right], t\right) \in T_{\mathcal{U}[N]}$. Now, since $h_{n+1}$ has to make the diagram commute, $h_{n+1}\left(t_{1}\right)$ must be of the form $(B, t)$ and, since it has to be a morphism, it must be $\partial_{u[N]}^{0}((B, t))=\bigoplus B=h_{n+1}\left(\partial_{\Theta}^{0}\left(t_{1}\right)\right)$. Therefore $h_{n+1}\left(t_{1}\right)=t_{0}$. Observe that there is only one choice for $h_{n+1}\left(t_{1}\right)$, given $k$ and $h_{n}$ by inductive hypothesis. Obviously, $\varepsilon_{N} \circ h_{n+1}^{+}\left(t_{1}\right)=t=$ $k\left(t_{1}\right)=k \circ \mu_{n+1}^{+}\left(t_{1}\right)$. Now, let $\partial_{\Theta^{+}}^{1}\left(t_{1}\right)=\bigoplus_{i} n_{i} a_{i}$. So $\partial_{\Theta}^{1}\left(t_{1}\right)=\bigoplus_{i}\left(\bigoplus\left\{a_{i}\right\} \times\left[n_{i}\right]\right)$ in $\Theta$. Suppose that $k\left(a_{j}\right)=\bigoplus_{l} m_{l}^{j} b_{l}^{j}$. By definition of $k$, since it does not merge different places in the post-set of a transition and $k\left(\partial_{\Theta^{+}}^{1}\left(t_{1}\right)\right)=\partial_{N}^{1}\left(k\left(t_{1}\right)\right)$, we have $\partial_{N}^{1}\left(k\left(t_{1}\right)\right)=v \oplus \bigoplus_{l} n_{j} m_{l}^{j} b_{l}^{j}$, with $\left.b_{l}^{j} \notin[v]\right]$. Thus in $\mathcal{u}[N]$ we have the places $\bigcup_{l}\left\{\left(\left\{t_{0}\right\}, b_{l}^{j}\right)\right\} \times\left[n_{j} m_{l}^{j}\right]$. We define

$$
h_{n+1}\left(a_{j}, i\right)=\bigoplus_{l}\left(\bigoplus\left\{\left(\left\{t_{0}\right\}, b_{l}^{j}\right)\right\} \times\left[m_{l}^{j}\right]_{i}\right) .
$$

So $\quad h_{n+1}^{+}\left(a_{j}\right)=\left(h_{n+1}\left(a_{j}, i\right)\right)^{+}=\bigoplus_{l} m_{l}^{j}\left(\left\{t_{0}\right\}, b_{l}^{j}\right)$ and

$\varepsilon_{N} \circ h_{n+1}^{+}\left(a_{j}\right)=\bigoplus m_{l}^{j} b_{l}^{j}=k\left(a_{j}\right)=k \circ \mu_{n+1}^{+}\left(a_{j}\right)$.

Observe that $h_{n+1}\left(a_{j}, i\right)$ is completely determined by $k$ and by the conditions of decorated occurrence net morphisms.

Finally, we have to show that $h_{n+1}$ is a morphism $\Theta^{(n+1)} \rightarrow \mathfrak{u}[N]$. But this task is really trivial because, by its own construction, $h_{n+1}$ preserves source, target and initial marking and respects families.

Theorem 4.8. (Correspondence with Winskel's Safe Net Unfoldings (Winskel 1986)) Let $N$ be a semiweighted net. Then, the unfolding $\mathcal{u}[N]$ is (isomorphic to) an occurrence net and therefore, by Proposition 3.9, $\mathcal{u}[N]^{+} \cong \mathcal{U}[N]$. Moreover, if $N$ is a safe net, $\mathcal{U}[N]$ is (isomorphic to) Winskel's unfolding of $N$. Finally, whenever $N$ is (isomorphic to) an occurrence net, the unit of the adjunction $\left\langle\left({ }_{-}\right)^{+}, \mathcal{U}[-]\right\rangle:$ DecOcc $\rightarrow$ PTNets, $\eta_{N}: N \rightarrow \mathcal{U}\left[N^{+}\right] \cong \mathcal{U}[N]$, is an isomorphism. Therefore, $\left\langle\left({ }_{-}\right)^{+}, \mathcal{U}[-]\right\rangle$ restricts to a coreflection $\langle\hookrightarrow, \mathcal{U}[-]\rangle:$ Occ $\rightarrow$ SWNets and to Winskel's coreflection $\langle\hookrightarrow, \mathfrak{u}[-]\rangle:$ Occ $\rightarrow$ Safe.

Proof. Concerning the claimed correspondence, it is enough to observe that, when $N$ is safe, our definition of $\mathcal{U}[N]$ is such that $(b, 1)$ is a place in $\mathcal{U}[N]$ if and only if $b$ is a condition in Winskel's unfolding. So $u[N]^{+}$and $\varepsilon_{N}$ are exactly Winskel's unfolding and folding morphism for $N$. The other statements are evident.

Observe that the coreflection between Occ and SWNets allows us to extend immediately 
Winskel's chain of coreflections to SWNets, namely by composition with the coreflections between Dom and PES and between PES and Occ. The following example shows the semantics associated by such a chain to a simple, well-known, non-safe semiweighted net.

\section{Example 4.9.}

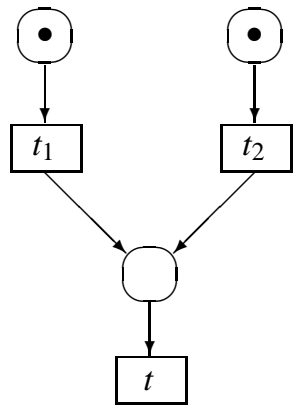

$N$
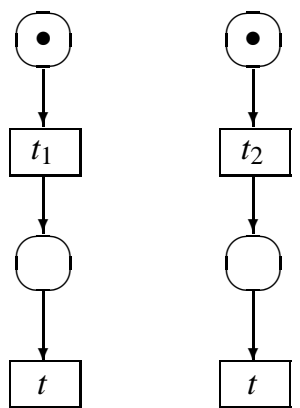

$\mathcal{U}[N]$

Observe that the unfolding contains two concurrent copies of $t$. These correspond to the occurrences of $t$ in two possible 'causal contexts', namely $t$ caused by $t_{1}$ and $t$ caused by $t_{2}$. In the picture below, which shows the event structure and the prime algebraic domain associated to $N$, the four events so arising are labelled by the transition they correspond to.

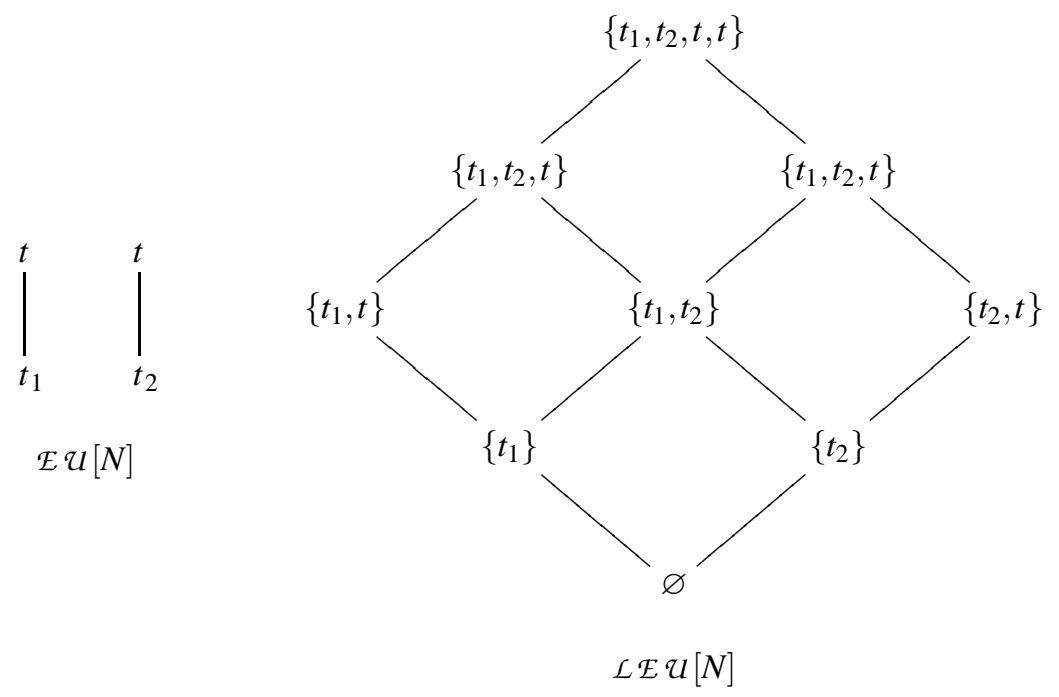

\section{PT Nets, Event Structures and Domains}

In this section, we show an adjunction between occurrence nets and decorated occurrence nets. Composing this adjunction with that given in Section 4, we obtain an adjunction between Occ and PTNets. Moreover, exploiting Winskel's coreflections in (Winskel 1986), we obtain adjunctions between PES and PTNets and between Dom and PTNets, as explained in the Introduction.

We first define a functor from decorated occurrence nets to occurrence nets. It is simply the forgetful functor which forgets about the structure of families. 
Definition 5.1. ( $\mathcal{F}[]]$ : from DecOcc to Occ) Given a decorated occurrence net $\Theta$ define $\mathcal{F}[\Theta]$ to be the occurrence net $\Theta$. Furthermore, given $\langle f, g\rangle: \Theta_{0} \rightarrow \Theta_{1}$, define $\mathcal{F}[\langle f, g\rangle]$ to be $\langle f, g\rangle$.

In order to define a left adjoint for $\mathcal{F}[]$, we need to identify, for each occurrence net $\Theta$, a decorated occurrence net $\mathcal{D}[\Theta]$ which is, informally speaking, a 'place-saturated' version of $\Theta$, in the sense that, whilst exhibiting the same behaviour as $\Theta$, it contains exactly enough families to match in a unique way the structure of any decorated occurrence net whose transitions are 'similar' to those of $\Theta$. More precisely, the existence of an adjunction requires $\mathcal{D}[\Theta]$ to be such that, for any occurrence net $\Theta^{\prime}$,

$$
\operatorname{Occ}\left[\Theta, \mathcal{F}\left[\Theta^{\prime}\right]\right] \cong \operatorname{DecOcc}\left[\mathcal{D}[\Theta], \Theta^{\prime}\right]
$$

i.e., the set of morphisms from $\Theta$ to $\mathcal{F}\left[\Theta^{\prime}\right]$ in Occ and the set of morphisms from $\mathcal{D}[\Theta]$ to $\Theta^{\prime}$ in DecOcc are isomorphic. It follows from this condition that each transition of $\mathcal{D}[\Theta]$ must have enough families in its post-set to 'cover' those in the post-set of any transition of $\Theta^{\prime}$ to which it could be mapped by an occurrence net morphism and, at the same time, it must not have too many of them so that such a covering is realized by a unique decorated occurrence net morphism from $\mathcal{D}[\Theta]$ to $\Theta^{\prime}$.

Because of the uniqueness requirement, saturating occurrence nets is a delicate matter: we need to identify a suitable set of families which can 'represent' uniquely all the possible others. To this aim are devoted the following definition and lemma, where the relation $\mapsto$ is introduced to capture the behaviour of decorated occurrence net morphisms on families-which will be represented as strings on appropriate alphabets—and prime strings are meant to represent-in a sense that will be clear later—exactly the families which we must add to $\Theta$ in order to saturate it.

In the following, given a string $s$ on an alphabet $\Sigma$, we denote the $i$ th element of $s$ by $s_{i}$ and its length by $|s|$. We shall use $\sigma_{i}, i \in \omega$, to range over $\Sigma$. For $n \in \omega, \sigma_{i}^{n}$ will denote the string consisting of the symbol $\sigma_{i}$ repeated $n$ times. Writing a string $s$ as $\sigma_{1}^{n_{1}} \cdots \sigma_{k}^{n_{k}}$ we imply that $\sigma_{i} \neq \sigma_{i+1}, i=1, \ldots, k-1$.

Definition 5.2. (Prime Strings) Let $\Sigma$ be an alphabet, i.e., a set of symbols. Define the binary relation $\mapsto$ on $\Sigma^{+}$, the language of non-empty strings on $\Sigma$, by

$$
\sigma_{1}^{n_{1}} \cdots \sigma_{k}^{n_{k}} \mapsto \sigma_{1}^{m_{1}} \cdots \sigma_{k}^{m_{k}} \quad \Longleftrightarrow \quad \exists q \in \omega, q n_{i}=m_{i}, i=1, \ldots, k .
$$

Define the language of prime strings on $\Sigma$ to be

$$
\Sigma^{P}=\Sigma^{+}-\left\{\sigma_{1}^{n_{1}} \sigma_{2}^{n_{2}} \cdots \sigma_{k}^{n_{k}} \mid \sigma_{i} \in \Sigma, \operatorname{gcd}\left(n_{1}, \ldots, n_{k}\right)>1\right\},
$$

where gcd is the greatest common divisor.

Lemma 5.3. (Prime Strings are primes) Given $s^{\prime} \in \Sigma^{+}$there exists a unique $s \in \Sigma^{P}$ such that $s \mapsto s^{\prime}$.

Proof. Let $s^{\prime}=\sigma_{1}^{m_{1}} \cdots \sigma_{k}^{m_{k}}$, where $\sigma_{i} \neq \sigma_{i+1}$. Consider $h=\operatorname{gcd}\left(m_{1}, \ldots, m_{k}\right)$. Since $h$ is the unique integer such that $m_{i}$ is divisible by $h$ for $1 \leq i \leq k$ and $\operatorname{gcd}\left(\frac{m_{1}}{h}, \ldots, \frac{m_{k}}{h}\right)=1$, and since $h$ always exists (possibly $h=1$ ) we have that $s=\sigma_{1}^{m_{1} / h} \cdots \sigma_{k}^{m_{k} / h}$ is the unique prime string such that $s \mapsto s^{\prime}$.

We start relating strings and nets by looking at sets of places as alphabets and at families as strings on such alphabets. Given a (decorated) occurrence net $\Theta$ and a transition $t \in T_{\Theta}$, we 
denote by $\Sigma_{\{t\}}$ the alphabet $\left[\left[\partial_{\Theta}^{1}(t)\right]\right]$. By analogy, since the places in the initial marking are in the post-set of no transition, $\Sigma_{\varnothing}$ will consist of the places $\left[\left[u_{\Theta}\right]\right]$; following the analogy, in the rest of the section $u_{\Theta}$ will also be denoted by $\partial_{\Theta}^{1}(\varnothing)$.

Since a family $b^{F}$ of a decorated occurrence net $\Theta$ is nothing but an ordered subset of the initial marking or of the post-set of a transition, it corresponds naturally to a string in $\Sigma_{x}^{+}$where $x=\bullet\left[b^{F}\right]$, namely, the string of length $\left|\left[\left[b^{F}\right]\right]\right|$ whose $i$ th element is $(b, i)$. We will write $\bar{b}^{F}$ to indicate such a string.

Now, we can define the saturated net corresponding to an occurrence net $\Theta$. It is the net $\mathcal{D}[\Theta]$ whose transitions are the transitions of $\Theta$, and whose families in the post-set of a transition $t$ are the prime strings on the alphabets defined by $t$ in $\Theta$. It is immediate to see that this construction is well-defined, i.e., that $\mathcal{D}[\Theta]$ is a decorated occurrence net.

Definition 5.4. ( $\mathcal{D}[-]$ : from Occ to $\mathrm{DecOcc}$ ) Let $\Theta$ be a net in Occ. We define the decorated occurrence net $\mathcal{D}[\Theta]$ to be the net $\left(\partial_{\mathcal{D}[\Theta]}^{0}, \partial_{\mathcal{D}[\Theta]}^{1}:\left(T_{\Theta}, 0\right) \rightarrow S_{\mathcal{D}[\Theta]}^{\mathcal{M}}, u_{\mathcal{D}[\Theta]}\right)$, where

$-S_{\mathcal{D}[\Theta]}=\bigcup\left\{\{s\} \times[|s|] \mid s \in \Sigma_{x}^{P}\right.$ and $\left(x=\{t\} \subseteq T_{\Theta}\right.$ or $\left.\left.x=\varnothing\right)\right\}$

$\left.-\partial_{\mathcal{D}[\Theta]}^{0}(t)=\bigoplus\left\{(s, i) \in S_{\mathcal{D}[\Theta]} \mid s_{i} \in\left[\partial_{\Theta}^{0}(t)\right]\right]\right\}$;

$-\partial_{\mathcal{D}[\Theta]}^{1}(t)=\bigoplus\left\{(s, i) \in S_{\mathcal{D}[\Theta]} \mid s_{i} \in\left[\left[\partial_{\Theta}^{1}(t)\right]\right\}=\bigoplus\left\{s^{F} \mid s \in \Sigma_{\{t\}}^{P}\right\} ;\right.$

$-u_{\mathcal{D}[\Theta]}=\bigoplus\left\{s^{F} \mid s \in \Sigma_{\varnothing}^{P}\right\}$.

Example 5.5.

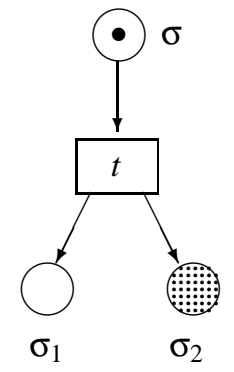

$\Theta$

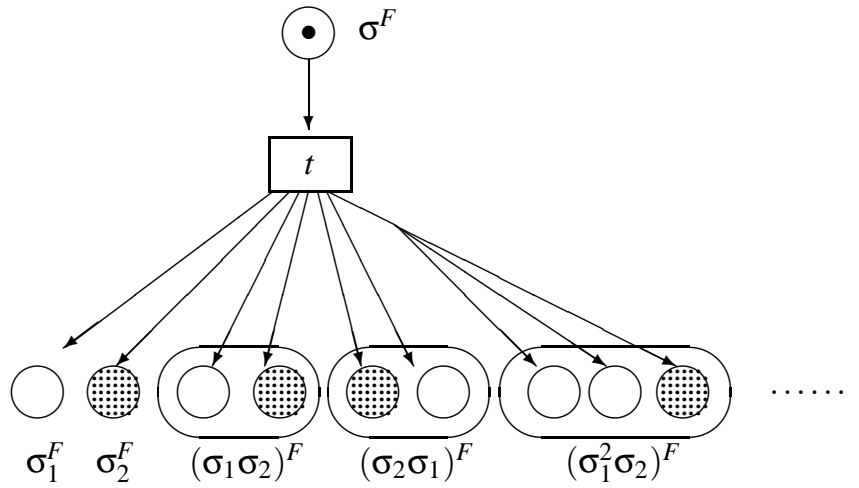

$\mathcal{D}[\Theta]$

An occurrence net $\Theta$ and (part of) the decorated occurrence net $\mathcal{D}[\Theta]$

We now select a candidate for the unit of the adjunction.

Proposition 5.6. (Unit Morphism) Given an occurrence net $\Theta$ consider the map $\eta_{\Theta}: \Theta \rightarrow$ $\mathcal{F} \mathcal{D}[\Theta]$ defined by

$$
\begin{aligned}
& \eta_{\Theta}(t)=t \\
& \eta_{\Theta}(a)=\bigoplus\left\{(s, i) \in S_{\mathcal{D}[\Theta]} \mid s_{i}=a\right\} .
\end{aligned}
$$

Then $\eta_{\Theta}$ is a morphism in Occ.

Proof. The non-trivial case is that of condition $(i v)$ in the definition of morphisms: 


$$
\begin{aligned}
\eta_{\Theta}\left(\partial_{\Theta}^{i}(t)\right) & =\bigoplus\left(\left\{\eta_{\Theta}(a) \mid a \in \llbracket\left[\partial_{\Theta}^{i}(t) \rrbracket\right\}\right)\right. \\
& =\bigoplus\left\{(s, i) \mid s_{i}=a \text { and } a \in \llbracket \llbracket \partial_{\Theta}^{i}(t) \rrbracket\right\}=\partial_{\mathscr{F} \mathcal{D}[\Theta]}^{i}(t) .
\end{aligned}
$$

In order to illustrate the above definition, considering again the net $\Theta$ of Example 5.5. For such a net we have that

$$
\begin{aligned}
& \eta_{\Theta}\left(\sigma_{1}\right)=\left(\sigma_{1}, 1\right) \oplus\left(\sigma_{1} \sigma_{2}, 1\right) \oplus\left(\sigma_{2} \sigma_{1}, 2\right) \oplus\left(\sigma_{1}^{2} \sigma_{2}, 1\right) \oplus\left(\sigma_{1}^{2} \sigma_{2}, 2\right) \oplus \cdots ; \\
& \eta_{\Theta}\left(\sigma_{2}\right)=\left(\sigma_{2}, 1\right) \oplus\left(\sigma_{1} \sigma_{2}, 2\right) \oplus\left(\sigma_{2} \sigma_{1}, 1\right) \oplus\left(\sigma_{1}^{2} \sigma_{2}, 2\right) \oplus \cdots .
\end{aligned}
$$

Before showing that $\eta_{\Theta}$ is universal, we need to develop further the relation between nets and strings. Since a morphism maps post-sets to post-sets, it naturally induces a (contravariant) mapping between the languages associated to transitions related by the morphism. To simplify the exposition, in the rest of this section, for $k$ a morphism of nets, $k(\{t\})$ and $k(\varnothing)$, denote, respectively, $\{k(t)\}$ and $\varnothing$; moreover, $\partial_{\Theta}^{1}(\{t\})$ denotes $\partial_{\Theta}^{1}(t)$.

Definition 5.7. ( $S_{k}^{x}$ : from $\Sigma_{k(x)}^{+}$to $\Sigma_{x}^{+}$) Let $\Theta_{0}$ and $\Theta_{1}$ be (decorated) occurrence nets, let $k=$ $\langle f, g\rangle: \Theta_{0} \rightarrow \Theta_{1}$ be a morphism and let $x=\{t\} \subseteq T_{\Theta_{0}}$ or $x=\varnothing$ and $y$ be such that $f(x)=y$. Then $k$ induces a unique semigroup homomorphism $\mathcal{S}_{k}^{x}$ from $\Sigma_{y}^{+}$to $\Sigma_{x}^{+}$defined on the generators $b \in\left[\left[\partial_{\Theta_{1}}^{1}(y)\right]\right]$ by

$$
\left.S_{k}^{x}(b)=a \text { such that } a \in\left[\partial_{\Theta_{0}}^{1}(x)\right]\right] \text { and } g(a)=b .
$$

It follows from the properties of safe net morphisms in Proposition 1.5 that $\mathcal{S}_{k}^{x}$ is well-defined, i.e., there exists one and only one $a \in\left[\left[\partial_{\Theta_{0}}^{1}(x)\right]\right]$ such that $g(a)=b$.

To clarify the relation between $\mapsto$ and decorated occurrence net morphisms, observe that, in the condition of the previous definition, if $\Theta$ is a decorated occurrence net and $k$ is a decorated occurrence net morphism, then $\bar{a}^{F} \mapsto \mathcal{S}_{k}^{x}\left(\bar{b}^{F}\right)$ if and only if $k\left(a^{F}\right)=b^{F}$.

Example 5.8. Consider the nets in the following figure, where the morphism $\langle f, g\rangle$ is such that $g\left(\sigma_{1}\right)=s_{2} \oplus s_{3}$ and $g\left(\sigma_{2}\right)=s_{1}$.

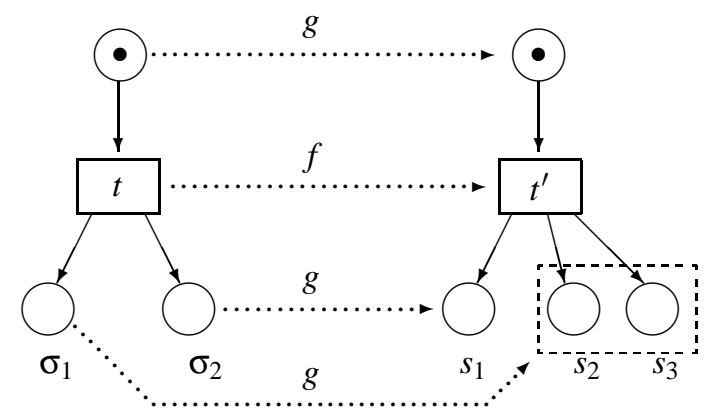

Then, for instance, we have that $\mathcal{S}_{\langle f, g\rangle}^{\{t\}}\left(s_{1} s_{2} s_{3} s_{2} s_{1}\right)=\sigma_{2} \sigma_{1}^{3} \sigma_{2}$.

Finally, we show that $\mathcal{D}$ [-] extends to a functor which is left adjoint to $\mathcal{F}[-]$.

Theorem 5.9. $(\mathcal{D}[-] \dashv \mathcal{F}[-])$ The pair $\langle\mathcal{D}[-], \mathcal{F}[-]\rangle:$ Occ $\rightarrow$ DecOcc constitutes an adjunction.

Proof. Let $\Theta$ be an occurrence net. By (MacLane 1971, Theorem 2, pg. 81) it is enough to show that the morphism $\eta_{\Theta}: \Theta \rightarrow \mathcal{F} \mathcal{D}[\Theta]$ is universal from $\Theta$ to $\mathcal{F}$, i.e., for any decorated occurrence net $\Theta^{\prime}$ and any $k: \Theta \rightarrow \mathcal{F}\left[\Theta^{\prime}\right]$ in Occ, there exists a unique $\langle f, g\rangle: \mathcal{D}[\Theta] \rightarrow \Theta^{\prime}$ in DecOcc such that 
$k=\mathcal{F}[\langle f, g\rangle] \circ \eta_{\Theta}$.

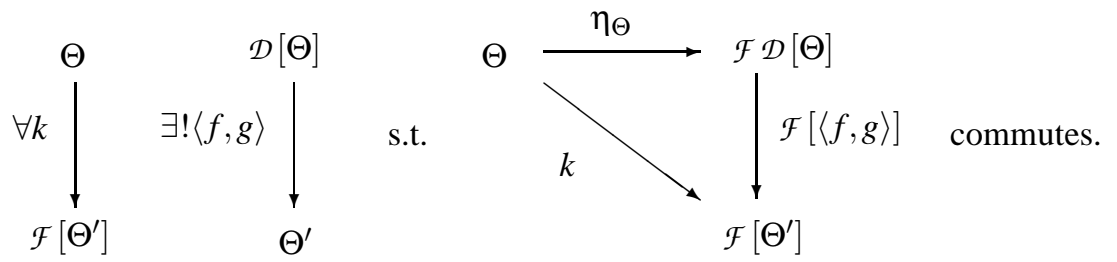

Given $\Theta^{\prime}$ and $k$, we define $\langle f, g\rangle: \mathcal{D}[\Theta] \rightarrow \Theta^{\prime}$ as follows:

$$
\begin{aligned}
& f(t)=k(t) \\
& \left.\llbracket b^{F}\right] \subseteq \llbracket\left[g\left(s^{F}\right)\right] \quad \Longleftrightarrow \quad s \mapsto S_{k}^{x}\left(\bar{b}^{F}\right), \text { where } x=\bullet\left[s^{F} \rrbracket \text { and } k(x)=\bullet \llbracket b^{F}\right]
\end{aligned}
$$

First remark that $\langle f, g\rangle$ is well-defined: if $s=\sigma_{1}^{n_{1}} \cdots \sigma_{r}^{n_{r}} \mapsto \mathcal{S}_{k}^{x}\left(\bar{b}^{F}\right)$ then there is one and only one way to have $\left[b^{F}\right] \subseteq\left[\left[g\left(s^{F}\right)\right]\right.$, namely

$$
g(s, i)=\bigoplus\{b\} \times[q]_{i},
$$

where $q$ is the unique integer such that $\sigma_{1}^{q n_{1}} \cdots \sigma_{r}^{q n_{r}}=S_{k}^{x}\left(\bar{b}^{F}\right)$.

Let $x=\left\{t_{0}\right\}$ or $x=\varnothing$. Observe that $\left.\forall a \in\left[\partial_{\Theta^{\prime}}^{1}(x)\right]\right]$

$$
\forall(b, j) \in\left[[k(a)] \exists !(s, i) \text { such that }(s, i) \in\left[\partial_{\mathscr{D}[\Theta]}^{1}(x) \rrbracket \text { and }(b, j) \in \llbracket g(s, i)\right] .\right.
$$

Moreover, $(s, i)$ is the unique place in $\mathcal{D}[\Theta]$ such that $s_{i}=a$ and $\left.(b, j) \in[g(s, i)]\right]$. In fact, for $x={ }^{\bullet} a$, by Lemma 5.3, there exists a unique $s \in \Sigma_{x}^{P}$ such that $s \mapsto \mathcal{S}_{k}^{x}\left(\bar{b}^{F}\right)$. If $(b, j) \in \llbracket k(a) \rrbracket$ then, since $k$ is a morphism, $k(x)=\bullet\left[b^{F}\right]$ and so there exists a unique $s^{F}$ in $\partial_{\mathscr{D}[\Theta]}^{1}(x)$ such that $\llbracket\left[b^{F}\right] \subseteq \llbracket g\left(s^{F}\right) \rrbracket$, i.e., $\exists !(s, i) \in \llbracket \partial_{\mathscr{D}[\Theta]}^{1}(x) \rrbracket$ such that $\left.(b, j) \in \llbracket g(s, i)\right]$. Obviously $s_{i}=a$, by definition of $\mathcal{S}_{k}^{x}$ and $\mapsto$. Moreover, if there were another such $\left(s^{\prime}, j\right)$, then $s^{\prime} \in \Sigma_{x}^{P}$ since $a$ belongs only to $\Sigma_{x}$. So by the previous lemma $s^{\prime}=s$ and, since $g$ respects families, $j=i$.

Now, if $(b, j) \in \llbracket g(s, i)]$, then $s \mapsto S_{k}^{x}\left(\bar{b}^{F}\right)$ and, therefore, $\mathcal{S}_{k}^{x}\left(\bar{b}^{F}\right)_{(i-1) q+1}, \ldots, \mathcal{S}_{k}^{x}\left(\bar{b}^{F}\right)_{i q}=s_{i}$, by definition of $\mapsto$. Thus, by definition of $\left.S_{k}^{x},\{b\} \times[q]_{i} \subseteq\left[k\left(s_{i}\right)\right]\right]$. So we have $\bigcup\left\{[g(s, i)] \mid s_{i}=\right.$ $a\}=[[k(a)]$. Obviously, all the $\llbracket[g(s, i)]]$ are disjoint and $\bigoplus[g(s, i) \rrbracket=g(s, i)$, since the families are disjoint. Therefore,

$$
\bigoplus\left\{g(s, i) \mid s_{i}=a\right\}=k(a) .
$$

It is now easy to see that the diagram commutes. For transitions this is clear. Concerning places, we have:

$$
\mathcal{F}[\langle f, g\rangle] \circ \eta_{\Theta}(a)=g\left(\bigoplus\left\{(s, i) \mid s_{i}=a\right\}\right)=k(a) .
$$

Now, consider any morphism $h: \mathcal{D}[\Theta] \rightarrow N$ which makes the diagram commute. Because of the definition of $\eta_{\Theta}$ on the transitions, $h$ must be of the form $\left\langle f, g^{\prime}\right\rangle$. We have to show that, necessarily $g=g^{\prime}$.

Let $\left[b^{F}\right] \subseteq\left[g^{\prime}\left(s^{F}\right)\right]$. So, the family of $b$ must be $\{b\} \times[q n]$ for some $q$, where $n=|s|$. Since $\left\langle f, g^{\prime}\right\rangle$ is a morphism, given $\left.x=\bullet\left[s^{F}\right]\right]$ and $y=\bullet\left[b^{F}\right]$, it must be $f(x)=y$. Since the diagram must commute and $s^{F}$ is the unique family in $\partial_{\mathscr{D}[\Theta]}^{1}(x)$ whose image contains $b^{F}$, it must be $\{b\} \times[q]_{i} \subseteq\left[k\left(s_{i}\right)\right]$ for $i=1, \ldots, n$. Therefore, we have $\mathcal{S}_{k}^{x}\left(\bar{b}^{F}\right)=s_{1}^{q} \cdots s_{n}^{q}$ and so $s \mapsto S_{k}^{x}\left(\bar{b}^{F}\right)$, 
which means, by definition of $g$, that $\left[\left[b^{F} \rrbracket \subseteq\left[g\left(s^{F}\right)\right]\right.\right.$. Hence, we have $\llbracket\left[g^{\prime}\left(s^{F}\right)\right] \subseteq\left[g\left(s^{F}\right) \rrbracket\right.$. On the other hand, suppose $\left.\llbracket b^{F}\right] \subseteq \llbracket\left[g\left(s^{F}\right)\right]$. Then $s \mapsto \mathcal{S}_{k}^{x}\left(\bar{b}^{F}\right)$, for some $x$. Necessarily, it must exist $s^{\prime F}$ with $s^{\prime} \in \Sigma_{x}^{P}$ such that $\left[b^{F}\right] \subseteq\left[g^{\prime}\left(s^{\prime F}\right)\right]$. Then, by Lemma 5.3, such an $s^{\prime}$ cannot be anything but $s$. Therefore $\left[g\left(s^{F}\right)\right] \subseteq\left[g^{\prime}\left(s^{F}\right)\right]$ and, reasoning as before, $g^{\prime}\left(s^{F}\right)=g\left(s^{F}\right)$. We can then conclude that $g^{\prime}=g$ and $h=\langle f, g\rangle$.

Let us now show that $\langle f, g\rangle$ is a morphism. It is enough to verify conditions $i$ and $i i$ in Proposition 1.5. Let $x=\left\{t_{0}\right\}$ or $x=\varnothing$ and $f(x)=y$. If $\left[b^{F}\right] \subseteq\left[g\left(\partial_{\mathcal{D}[\Theta]}^{1}(x)\right)\right]$, then by definition of $g$, we have $\left[b^{F}\right] \subseteq\left[\llbracket \partial_{\Theta^{\prime}}^{1}(y)\right]$. So $\left[g\left(\partial_{\mathcal{D}[\Theta]}^{1}(x)\right) \rrbracket \subseteq \llbracket \partial_{\Theta^{\prime}}^{1}(y)\right]$. Observe that this, together with property (1), proves the required conditions both on the initial marking and on $\partial_{\mathscr{D}[\Theta]}^{1}$. We still have to check that $\langle f, g\rangle$ respects sources.

Suppose $f\left(t_{0}\right)=t_{1}$. Let $\left.(s, i) \in\left[\partial_{\mathcal{D}[\Theta]}^{0}\left(t_{0}\right)\right]\right]$ and $(b, j) \in\left[\left[g(s, i) \rrbracket\right.\right.$. Then $\left.\left.s_{i} \in \llbracket \partial_{\Theta}^{0}\left(t_{0}\right)\right]\right]$ and since $\bigoplus\left\{g(s, i) \mid s_{i}=a\right\}=k(a)$, we have $\left.(b, j) \in\left[k\left(s_{i}\right)\right] \subseteq\left[\partial_{\Theta^{\prime}}^{0}\left(t_{1}\right)\right]\right]$, since $k$ is a morphism and $k\left(t_{0}\right)=t_{1}$. So $\left.\llbracket g\left(\partial_{\mathcal{D}[\Theta]}^{0}\left(t_{0}\right)\right)\right] \subseteq\left[\partial_{\Theta^{\prime}}^{0}\left(f\left(t_{0}\right)\right)\right]$. Now, if $(b, j) \in\left[\partial_{\Theta^{\prime}}^{0}\left(t_{1}\right)\right]$ then there exists a unique $a \in\left[\left[\partial_{\Theta}^{0}\left(t_{0}\right)\right]\right]$ such that $(b, j) \in[k k(a)]$. Therefore, there exists a unique $(s, i)$ such that $(b, j) \in$ $\llbracket\left[g(s, i) \rrbracket\right.$ and $s_{i}=a$. Thus, $\left.(s, i) \in \llbracket \partial_{\mathcal{D}[\Theta]}^{0}\left(t_{0}\right)\right]$. Now, if $\left(s^{\prime}, j\right) \in\left[\partial_{\mathcal{D}[\Theta]}^{0}\left(t_{0}\right)\right]$ is such that $(b, j) \in$ $\llbracket g\left(s^{\prime}, j\right) \rrbracket$ it must be $s_{j}^{\prime}=a$, otherwise $a$ would not be the unique element in $\left.\llbracket \partial_{\Theta}^{0}\left(t_{0}\right)\right]$ whose image contains $(b, j)$. Therefore, $\left(s^{\prime}, j\right)=(s, i)$.

The next corollary summarizes the results we obtain via the adjunctions $\langle\mathcal{D}[$ [-], $\mathcal{F}[-]\rangle:$ Occ $\rightarrow$ DecOcc and $\left\langle\left({ }_{-}\right)^{+}, \mathfrak{u}[-]\right\rangle:$ DecOcc $\rightarrow$ PTNets introduced here, and via Winskel's coreflections $\langle\mathcal{N}[-], \mathcal{E}[-]\rangle:$ PES $\rightarrow$ Occ and $\langle\operatorname{Pr}[-], \mathcal{L}[-]\rangle:$ Dom $\rightarrow$ PES.

Corollary 5.10. (Extensions of Winskel's coreflections (Winskel 1986)) The following are adjunctions whose right adjoints relate PT nets to, respectively, occurrence nets, prime event structures and prime algebraic domains.

$\left.-\left\langle()_{-}\right)^{+} \mathcal{D}[-], \mathcal{F} \mathfrak{U}[-]\right\rangle:$ Occ $\rightarrow$ PTNets;

$-\left\langle(-)^{+} \mathcal{D} \mathcal{N}[-], \mathcal{E} \mathcal{F} \mathcal{U}[-]\right\rangle:$ PES $\rightarrow$ PTNets;

$-\left\langle()^{+} \mathcal{D} \mathcal{N} \mathcal{P} r[], \mathcal{L E F} \mathcal{U}[-]\right\rangle:$ Dom $\rightarrow$ PTNets.

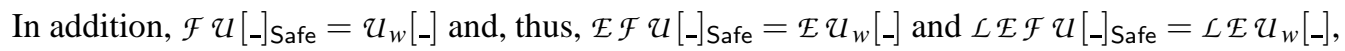
i.e., the semantics given to safe nets by the chain of adjunctions presented in this work coincides with the semantics given to them by Winskel's chain of coreflections.

Also, the functors $\mathcal{F} \mathcal{U}[$ [] $:$ SWNets $\rightarrow$ Occ, $\mathcal{E} \mathcal{F} \mathcal{U}[$ [] $:$ SWNets $\rightarrow$ PES, and $\mathcal{L} \mathcal{E} \mathcal{F} \mathcal{U}[$ [] : SWNets $\rightarrow$ Dom admit coreflection left adjoints.

Finally, the following example shows the semantics associated to the net of Example 4.4 by the adjunction $\left\langle(-)^{+} \mathcal{D} \mathfrak{N}[-], \mathcal{E F} \mathcal{U}[-]\right\rangle$ 


\section{Example 5.11.}

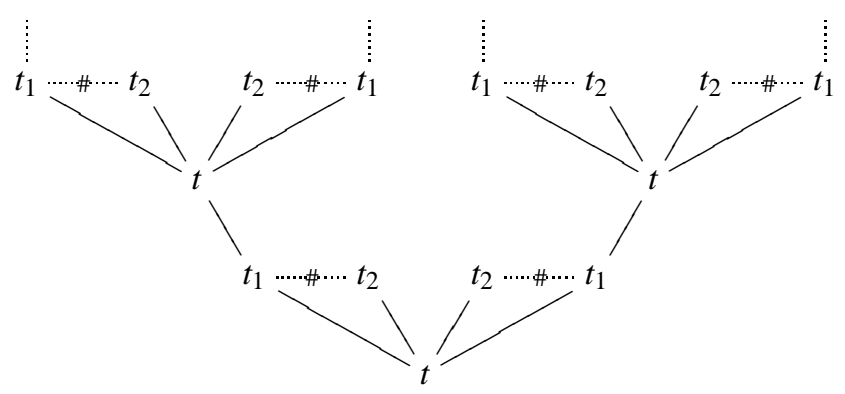

The event structure $\mathcal{E} \mathcal{F} \mathcal{U}[N]$ corresponding to the net $N$ of Example 4.4

\section{Conclusion and Related Work}

In this paper we have presented an extension to the case of PT nets of Winskel's semantics for safe nets (Winskel 1986). This extended semantics is given by a chain of adjunctions between the categories of finitary prime algebraic domains, of prime event structures and of occurrence nets. These results have been achieved by identifying a suitable adjunction between the category of PT nets and the category of occurrence nets, and by exploiting the existing adjunctions between occurrence nets, prime event structures and prime algebraic domains.

As already mentioned in the introduction, our purpose here was to describe the behaviour of PT nets by means of prime event structures, i.e., at the level of abstraction at which the identity of every event occurring in a computation and its causal interactions with the other events are unambiguous. This yields an explanation of net behaviours in which causality is the primary interest.

The existence of left adjoints to the unfolding functors guarantees the universality of the constructions, which in turn means that our semantics is as good as possible, given the chosen categories. In the present context this is precisely the relevance we attribute to such functors. In particular, $\mathcal{D}[$ [- $]$ is a rather complicated construction. Nevertheless, we remark that the important fact remains that such a functor exists.

In (Winskel 1988), Winskel shows that, in the case of safe nets, the domain associated to $N$ via the unfolding construction can be equivalently obtained by unfolding $N$ to a Mazurkiewicz trace language (Mazurkiewicz 1988) whose alphabet consists exactly of the transitions of $N$. Such a construction is clearly not possible in the case of general PT nets; an immediate reason for that is provided by the autoconcurrency which PT nets can exhibit, and which cannot be handled properly by trace languages. On the other hand, it is equally clear that one can build such a trace language by choosing appropriately the underlying alphabet. However, it seems that a satisfactory solution to this issue has to deal with a generalization of trace languages, e.g. (Hoogers et al. 1992; Sassone et al. 1993b).

The adjunction between PTNets and Occ factorizes through two adjunctions involving a category of decorated occurrence nets, DecOcc. Although this factorization may at first seem to be just a convenient technical solution, there in fact are some insights on the semantics given by the present unfolding construction which can be gained by looking more closely at decorated occur- 
rence nets. In fact, the semantics we have introduced here is somehow close to both Goltz-Reisig processes (Goltz and Reisig 1983) and to concatenable processes (Degano et al. 1989), without coinciding precisely with either of them. In (Meseguer et al. 1994; Meseguer et al. 1996), the present authors give a process-oriented account of the unfolding construction. More precisely, we introduce a new notion of process, whose definition is suggested by the idea of families in decorated occurrence nets, and which are therefore called decorated processes, and we show that they capture the unfolding semantics, in the precise sense that there is a one-to-one translation between decorated processes of $N$ and finite configurations of $\mathcal{E F} \mathcal{U}[N]$. Then, following the approach of (Degano et al. 1989), we axiomatize the notion of decorated (concatenable) process in terms of monoidal categories. More precisely, we define an abstract symmetric monoidal category $\mathcal{D} P[N]$ and we show that its arrows represent decorated concatenable processes. Then, we have that the following diagram commutes (up to equivalence)

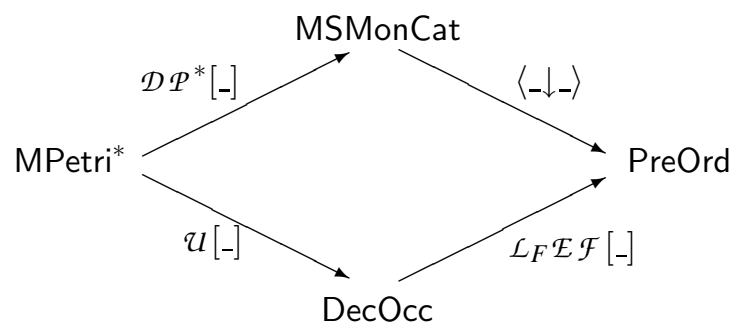

where MPetri* is the full subcategory of PTNets consisting of the nets with finite pre- and postsets, MSMonCat is the category of the 'marked' symmetric strict monoidal categories, i.e., symmetric strict monoidal categories $C$ with a distinguished object $c \in \mathrm{C}, \mathcal{D} \mathcal{P}^{*}[-]$ maps the marked net $\left(N, u_{N}\right)$ to $\left(u_{N}, \mathcal{D P}[N]\right)$, PreOrd is the category of preorders, $\left\langle_{-} \downarrow_{-}\right\rangle$is the comma category functor $(c, C) \mapsto\langle c \downarrow C\rangle$, and $L_{F}$ returns the finite configurations of prime event structures ordered by inclusion. We remark that an analogous result is proved in (Nielsen et al. 1990) in the case of elementary net systems - a particular class of safe nets without self-looping transitions.

We think that this is an interesting result, since it provides a unified account of the algebraic, the process-oriented, and the denotational views of net semantics. Moreover, it shows that decorated (deterministic) occurrence nets and their algebraic formalization in terms of monoidal categories, being the key to achieve such a unification, are structures of some relevance on their own.

To conclude, we would like to mention that the correspondence of semantics discussed above can be lifted smoothly to infinite computations. In (Sassone et al. 1993a), the present authors show that the symmetric monoidal category $\mathcal{P}[N]^{\omega}$ obtained as the completion of $\mathcal{P}[N]$ by colimits of $\omega$-diagrams can be understood as the category of possibly infinite concatenable processes of $N$. Working analogously, one can see that the arrows of the symmetric strict monoidal category $\mathcal{D P}[N]^{\omega}$ are possibly infinite decorated concatenable processes. Then, one can prove the commutativity (up to equivalence) of a diagram analogous to the one above involving all the configurations of $\mathcal{E} \mathcal{F} \mathcal{U}[N]$ and the comma category $\left\langle u_{N} \downarrow \mathcal{D} \mathcal{P}[N]^{\omega}\right\rangle$. The details of this construction can be found in (Sassone 1994). 


\section{References}

Best, E. and Devillers, R. (1987) Sequential and Concurrent Behaviour in Petri Net Theory. Theoretical Computer Science 55, 87-135.

Brown, C. and Gurr, D. (1990) A Categorical Linear Framework for Petri Nets. In Proceedings of the 5th LICS Symposium, 208-218, IEEE Press.

Brown, C., Gurr, D. and de Paiva, V. (1991) A Linear Specification Language for Petri Nets. Technical Report DAIMI PB-363, Computer Science Department, University of Aarhus, Aarhus, Denmark.

Cattani, G-L. and Sassone, V. (1996) Higher Dimensional Transition Systems. In Proceedings of the 11th LICS Symposium, IEEE Press, to appear.

Degano, P., De Nicola, R. and Montanari, U. (1988) A Distributed Operational Semantics for CCS based on Condition/Event Systems. Acta Informatica 26, 59-91.

Degano, P., Meseguer, J. and Montanari, U. (1989) Axiomatizing Net Computations and Processes. In Proceedings of the 4th LICS Symposium, 175-185, IEEE Press.

Engberg, U. and Winskel, G. (1993) Completeness Results for Linear Logic on Petri Nets. In Proceedings of MFCS 93, Lecture Notes in Computer Science 711, 442-452, Springer-Verlag.

Engelfriet, J. (1991) Branching Processes of Petri Nets. Acta Informatica 28, 575-591.

van Glabbeek, R. and Plotkin, G. (1995) Configuration Structures. In Proceedings of the 10th LICS Symposium, 293-307, IEEE Press.

van Glabbeek, R. and Vaandrager, F. (1987) Petri Net Models for Algebraic Theories of Concurrency. In Proceedings of PARLE, Lecture Notes in Computer Science 259, 224-242, Springer-Verlag.

Goubault, E. (1993) Domains of Higher Dimensional Automata. In Proceedings of CONCUR 93, Lecture Notes in Computer Science 720, 293-307, Springer-Verlag.

Goubault, E. and Jensen, T.P. (1992) Homology of Higher Dimensional Automata. In Proceedings of CONCUR 92, Lecture Notes in Computer Science 630, 254-268, Springer-Verlag.

Goltz, U. and Reisig, W. (1983) The Non-Sequential Behaviour of Petri Nets. Information and Computation 57, 125-147.

Gunawardena, J. (1993) A Generalized Event Structure for the Muller Unfolding of a Safe Net. In Proceedings of CONCUR 93, Lecture Notes in Computer Science 715, 278-292, Springer-Verlag.

Hoogers, P.W., Kleijn, H.C.M. and Thiagarajan, P.S. (1992) A Trace Semantics for Petri Nets. In Proceedings of ICALP 92, Lecture Notes in Computer Science 623, 595-604, Springer-Verlag.

Hoogers, P.W., Kleijn, H.C.M. and Thiagarajan, P.S. (1993) Local Event Structures and Petri Nets. In Proceedings of CONCUR 93, Lecture Notes in Computer Science 715, 462-476, Springer-Verlag.

MacLane, S. (1971) Categories for the Working Mathematician. Graduate Texts in Mathematics, SpringerVerlag.

Martí-Oliet, N. and Meseguer, J. (1991) From Petri Nets to Linear Logic through categories: A survey. International Journal of Foundations of Computer Science 2, 297-399.

Mazurkiewicz, A. (1988) Basic Notions of Trace Theory. In Lecture notes for the REX summerschool in temporal logic, Lecture Notes in Computer Science 354, 285-363, Springer-Verlag.

Meseguer, J. and Montanari, U. (1990) Petri Nets are Monoids. Information and Computation 88, 105-155.

Meseguer, J. Montanari, U. and Sassone, V. (1992) On the Semantics of Petri Nets. In Proceedings of CONCUR 92, Lecture Notes in Computer Science 630, 286-301, Springer-Verlag.

Meseguer, J. Montanari, U. and Sassone, V. (1994) On the Model of Computation of Place/Transition Petri Nets. In Proceedings of the 15th International Conference on Application and Theory of Petri Nets, Lecture Notes in Computer Science 815, 16-38, Springer-Verlag.

Meseguer, J. Montanari, U. and Sassone, V. (1996) Process versus Unfolding Semantics for Place/Transition Petri Nets. Theoretical Computer Science 153, 171-210.

Milner, R. (1989) Communication and Concurrency. Prentice-Hall. 
Mukund, M. (1992) Petri Nets and Step Transition Systems. International Journal of Foundations of Computer Science 3, 443-478.

Nielsen, M., Plotkin, G. and Winskel, G. (1981) Petri Nets, Event Structures and Domains, Part 1. Theoretical Computer Science 13, 85-108.

Nielsen, M., Rozenberg, G. and Thiagarajan, P.S. (1990) Behavioural Notions for Elementary Net Systems. Distributed Computing 4, 45-57.

Olderog, E.R. (1987) A Petri Net Semantics for CCSP. In Advances in Petri Nets, Lecture Notes in Computer Science 255, 196-223, Springer-Verlag.

Petri, C.A. (1962) Kommunikation mit Automaten. PhD thesis, Institut für Instrumentelle Mathematik, Bonn, Germany.

Petri, C.A. (1973) Concepts of Net Theory. In Proceedings of MFCS 73, Mathematics Institute of the Slovak Academy of Science, 137-146.

Petri, C.A. (1977) Non-Sequential Processes. Interner Bericht ISF-77-5, Gesellschaft für Mathematik und Datenverarbeitung, Bonn, Germany.

Pratt, V. (1991) Modeling Concurrency with Geometry. In Proceedings of POPL 91, 311-322, ACM Press.

Reisig, W. (1985) Petri Nets. Springer-Verlag.

Sassone, V., Meseguer, J. and Montanari, U. (1993a) $\omega$-Ind Completion of Monoidal Categories and Infinite Petri Net Computations. Presentation at the Workshop on Topology and Completion in Semantics, Institute Blaise Pascal, Chartres, France.

Sassone, V., Nielsen, M. and Winskel, G. (1993b) Deterministic Behavioural Models for Concurrency. In Proceedings of MFCS 93, Lecture Notes in Computer Science 711, 682-692, Springer-Verlag.

Sassone, V. (1994) On the Semantics of Petri Nets: Processes, Unfoldings, and Infinite Computations. PhD Thesis, TD 6/94 Dipartimento di Informatica, Università di Pisa, Italy.

Sassone, V. (1995) On the Category of Petri Net Computations. In Proceedings of TAPSOFT 95, Lecture Notes in Computer Science 915, 334-348, Springer-Verlag.

Sassone, V. (1996) An Axiomatization of the Algebra of Petri Net Concatenable Processes. Theoretical Computer Science, to appear.

Scott, D. (1970) Outline of a Mathematical Theory of Computation. In Proceedings of 4th Annual Princeton Conference on Information Science and Systems, 169-176.

Winskel, G. (1982) Event Structure Semantics for CCS and related languages. In Proceedings of the 9th ICALP, Lecture Notes in Computer Science 140, 561-576, Springer-Verlag.

Winskel, G. (1984) A New Definition of Morphism on Petri Nets. In Proceedings of STACS 84, Lecture Notes in Computer Science 166, 140-150, Springer-Verlag.

Winskel, G. (1986) Event Structures. In Proceedings of Advanced Course on Petri Nets, Lecture Notes in Computer Science 255, 325-392, Springer-Verlag.

Winskel, G. (1987) Petri Nets, Algebras, Morphisms and Compositionality. Information and Computation 72, 197-238.

Winskel, G. (1988) An Introduction to Event Structures. In Lecture notes for the REX summerchool in temporal logic, Lecture Notes in Computer Science 354, 365-397, Springer-Verlag. 